\title{
Cardinality versus Ordinality: A Suggested Compromise
}

\author{
By Michael MandleR*
}

\begin{abstract}
By taking sets of utility functions as primitive, we define an ordering over assumptions on utility functions that gauges their measurement requirements. Cardinal and ordinal assumptions constitute two levels of measurability, but other assumptions lie between these extremes. We apply the ordering to explanations of why preferences should be convex. The assumption that utility is concave qualifies as a compromise between cardinality and ordinality, while the Arrow-Koopmans explanation, supposedly an ordinal theory, relies on utilities in the cardinal measurement class. In social choice theory, a concavity compromise between ordinality and cardinality is also possible and rationalizes the core utilitarian policies. (JEL D01)
\end{abstract}

Ordinal utility theory permits only those assumptions on utility functions that are preserved under increasing transformations. The rationale for this rule is that any assumption $P$ not preserved under increasing transformations cannot be verified with observations of choice behavior: if a utility $u$ satisfies $P$, there will exist another utility $u^{\prime}$ that does not satisfy $P$ but that represents the same preferences as $u$. Nonordinal assumptions therefore seem to be needlessly restrictive: given a nonordinal assumption, one may always make a weaker assumption with the same implications for choice behavior. According to this view, the only role for cardinal utility is the normative one of representing interpersonal comparisons of utility.

Ordinalism's first targets were diminishing marginal utility (DMU) and concavity, which had long served as arguments for why consumer preferences should be convex. Neither DMU nor concavity is preserved by increasing transformations and hence neither is an ordinal assumption. The pioneer ordinalists such as Kenneth Arrow (1951) claimed that diminishing marginal utility is tantamount to assuming that utility is cardinal. Arrow's position remains predominant: either an assumption on utility is ordinal or it is cardinal (see, e.g., Andreu Mas-Colell et al., 1995, chap. 1).

\footnotetext{
* Department of Economics, Royal Holloway College, University of London, Egham, Surrey TW20 0EX, United Kingdom (e-mail: m.mandler@rhul.ac.uk). I thank Nick Baigent, Douglas Bernheim, Juan Dubra, Birgit Grodal, Duncan Luce, Roy Radner, Herbert Scarf, Phil Steitz, John Weymark, and an anonymous referee for many helpful comments, corrections, and suggestions.
}

This paper will take sets of utility functionswhich we call psychologies - as primitive; this will define a finer gradation of properties of utility that allows for intermediate standards of measurement. In this framework, ordinal utility theory takes the psychology consisting of all increasing transformations of any given utility function as primitive and lies at one end of the measurement spectrum. ${ }^{1}$ Cardinal utility theory takes the psychology consisting of all increasing affine transformations of a given utility as primitive; since this is a smaller set of utility functions, cardinality is a stronger (more restrictive) theory than ordinality. Ratio scales which take still smaller psychologies as primitive (the functions generated by all increasing linear transformations) are also common, particularly outside of economics. But in addition to these well-known measurement scales, there is an infinity of intermediate cases. Diminishing marginal utility and concavity lie precisely in the middle ground between cardinality and ordinality. The psychology consisting of all concave utility representations of a given preference relation is larger than any cardinal psychology it intersects, but smaller than any ordinal psychology it intersects. Concavity thus presupposes an intermediate standard of measurement and does not deserve its strongly nonordinalist reputation. Compared to a cardinal psychology, a concave psychology is easier to assemble in that an agent does not have to make as many utility

\footnotetext{
${ }^{1}$ We follow the convention of letting "the transformation $f$ of a function $u$ " refer to the composition $f \circ u$ rather $f$ itself.
} 
judgments. And concave psychologies are a natural primitive: they pinpoint the neoclassical principle that the value agents place on consumption increments progressively diminishes.

In preference theory, the main advantage of endowing agents with nonordinal properties of utility is that they can provide rationales for assumptions on preferences. Concavity and DMU form the leading examples and offer explanations of why and when preferences should be convex. To declare by fiat that preferences are convex (or that utility is quasiconcave) in contrast provides no rationale. That DMU lives on in textbooks as a motivation for convexity reflects the need for justification; but by relying on DMU, the textbook stories leave the measurement status of consumer theory up in the air. Textbooks in fact often suggest that DMU in one good does not affect the marginal utility of other goods, which amounts to an assumption of additive separability and hence of cardinality. In contrast, a concavity explanation of preference convexity incurs relatively small measurement cost.

In normative economics as well, a concavity compromise is possible. If interpersonal comparisons of utility are modeled with a set of concave utility functions, then one may still derive the plausible core of utilitarianism. Indeed, the policies backed by a concave psychology better approximate commonly held utilitarian intuitions than the policies derived from cardinal versions of utilitarianism. Moreover, due to their compromise status, concave interpersonal comparisons are less demanding than the cardinal comparisons that underlie traditional utilitarianism: fewer utility comparisons need to be made. We thus take issue with both prongs of the ordinalism-is-for-positive-theory versus cardinalismis-for-normative-theory dichotomy.

We argue that concavity can be tested observationally, although of course not using choice data alone. While economists once saw the Weber-Fechner law-that the marginal sensitivity to physical stimuli diminishes with stimulus intensity - as empirical support for DMU, the link to Weber-Fechner remained little more than an analogy. More recently, cognitive neuroscience and neuroeconomics have opened a new window on the physiological correlates of consumption decisions that suggests we may be able to compare a consumer's gains in satisfaction relative to a reference consumption level. As we will see, such a gauge would make it possible to test experimentally for DMU in consumption. Here, too, the existence of compromise measurement classes between ordinality and cardinality is relevant; the neural correlates of consumption decisions provide nonordinal information that may be insufficient to test for cardinality. Perhaps because of the cardinal versus ordinal gulf, neuroeconomics has not addressed such traditional economic topics as the measurement of utility. Compromise measurement categories may be able to reintegrate the study of consumer demand into a physiology and psychology of consumption.

The literature on self-reported happiness levels (e.g., Richard A. Easterlin, 1974; David G. Blanchflower and Andrew J. Oswald, 2004) would also gain from scrutiny of its measurement presuppositions. When the current literature judges a regression's goodness-of-fit as the sum of differences between reported and predicted contentment levels, it equates units of contentment and thereby treats well-being as a cardinal scale. One could instead judge fit by a compromise measurement standard in which all deviations of a subject's ranking of satisfaction increments from a predicted ranking would be treated as equal goodness-of-fit failures.

By taking sets of utility functions as primitive, we formalize what a nonordinal assumption on utility entails and judge just how nonordinal it is. To say simply that some agent's utility function satisfies diminishing marginal utility, for example, leaves open the question of exactly which utility functions accurately describe the agent's consumption experience. Cardinal understandings of diminishing marginal utility prior to the ordinal revolution would take a utility function satisfying DMU and all of its increasing affine transformations as primitive; all functions in this set would be taken as equivalent in every meaningful respect. But if one wishes to impose DMU and nothing more, then any utility that both exhibits DMU and represents the same preferences should be regarded as equally accurate. We therefore characterize the assumption of DMU using precisely this set of utility functions; in the same sense a cardinalist would, we take all these functions to be equivalent. This characterization allows us to gauge the extent of DMU's nonordinality and conclude that the assumption imposes relatively little measurement cost.

We begin the paper by showing that essentially any transitive preference relation $\succeq$ can be repre- 
sented by a psychology (even when $\succeq$ is incomplete). Then we order psychologies according to set inclusion. An ordering of assumptions on utility functions by set inclusion of the psychologies that satisfy those assumptions would not take us very far; it would apply only to trivial cases where one assumption implies another. But by requiring for a pair of assumptions that set inclusion holds only when a psychology has utilities that satisfy both assumptions simultaneously, we can avoid debilitating incompleteness, and measurement classes of assumptions can be calibrated finely. Claims that may appear nonsensical-e.g., "concavity" is a weaker assumption than "additive separability" — can be stated rigorously.

We then illustrate the ordering by comparing concavity with another celebrated rationale for the convexity of preferences, Arrow's (1951) argument (following Tjalling Koopmans) that an agent's leeway to determine the precise timing of consumption implies that preferences must be convex. Arrow reasoned that this rationale for convexity, unlike diminishing marginal utility, was free from any taint of cardinality. We show, however, that the utility theory that lies behind the ArrowKoopmans position is cardinal. The old neoclassical explanation, diminishing marginal utility or concavity, thus rests on less demanding measurement assumptions.

Next, we compare cardinality and concavity as a basis for utilitarianism. If a psychology consisting of concave interpersonally comparable utility functions is taken as primitive we can still derive the utilitarian recommendation that wealth be redistributed from rich to poor. The "concave utilitarianism" that results allows a tight derivation of commonly held utilitarian convictions, a goal that cardinal utilitarianism has notably failed to achieve. For example, concave utilitarianism characterizes the famous $\mathrm{Pi}$ gou-Dalton principle, which adds egalitarian constraints to a sum-of-cardinal-utilities ordering. Even Francis Y. Edgeworth (1897), the founding economic utilitarian, was suspicious of policy conclusions that relied on the cardinal details of a utilitarian social welfare function rather than on its concavity alone. Concave utilitarianism's better fit makes sense: it utilizes only the psychological premises that underlie neoclassical social welfare intuitions.

The standard cardinal formulation of utilitarianism requires an intimidating array of utility comparisons: a planner must post an exact rate at which gains in utility for one agent translate into gains for other agents. In contrast, for a concave utilitarian to dispense policy advice, all the planner must do is answer two questions affirmatively. First, is $j$ 's gain judged to be ordinally greater than $i$ 's loss? (The exact ratio of $j$ 's gain to $i$ 's loss is now irrelevant.) Second, is $i$ 's welfare level greater than $j$ 's? This recipe for concave utilitarian policies is similar to "poverty orderings" of income distributions, but via the device of least concave utility the recipe is applicable to agents who consume multiple goods. Concave utilitarianism's reliance on a more limited set of utility comparisons may be able to dampen the skepticism that surrounds social welfare judgments. The cardinal view demands hopelessly many interpersonal comparisons, encouraging the suspicion that those comparisons are wholly arbitrary and subjective.

We close by looking at how neural correlates of consumption can be used to test for concavity, and at how the present paper fits with earlier work on measurement.

\section{Psychologies and Orderings of Psychologies}

An agent will be represented by a set of utility functions $\mathbf{U}$, called a psychology, where each $u \in \mathbf{U}$ is defined on the same set $X$, which we call the domain of $\mathbf{U}$. A psychology lists the utility functions that as a set characterize the agent's psychological experience of consuming the bundles in $X$. Only those properties the utility functions in $\mathbf{U}$ have in common describe the agent's consumption experience. For example, suppose an agent has a psychology $\mathbf{U}$ containing every continuous function that represents some nontrivial preference relation on $\mathbb{R}^{n}$. This agent would then experience happiness or satisfaction as a continuous sensation, but an attribute of one of the utilities in $\mathbf{U}$ that is not shared by every other utility in $\mathbf{U}$ would not reflect the agent's attitudes toward consumption. One function in $\mathbf{U}$ might be concave, for example, but since there would then be nonconcave functions in $\mathbf{U}$ as well, this agent would not be characterized by the property of concavity. Psychologies can be assembled from several sources: choice behavior, physiological and psychological measurement (see Section V), agents' testimony (Section V), and introspection. 
Preference relations emerge straightforwardly from psychologies. We define $\succeq_{\mathbf{U}}$, the preferences induced by $\mathbf{U}$, by

$$
x \succeq_{\mathbf{U}} y \Leftrightarrow u(x) \geq u(y) \text { for all } u \in \mathbf{U} .
$$

We will refer interchangeably to $\succeq_{\mathbf{U}}$ satisfying an assumption on preferences and the underlying psychology $\mathbf{U}$ satisfying the same assumption.

Induced preferences can satisfy the assumptions usually imposed on preferences in economics. For instance, $\succeq_{\mathbf{U}}$ is complete if and only if, for all $u, v \in \mathbf{U}$ and $x, y \in A, u(x) \geq$ $u(y) \Leftrightarrow v(x) \geq v(y)$. For any psychology $\mathbf{U}, \succeq_{\mathbf{U}}$ must be transitive.

Since psychologies consist of sets of utility functions, they provide a more flexible tool for representing preferences than solitary utility functions. In fact, psychologies can induce almost any preference relation: except for transitivity and a technical requirement, any preference relation can be induced by some psychology, as Theorem 1 reports. We do not pursue the details, but we could generalize psychologies to cover even intransitive preferences by dropping the requirement that all functions in $\mathbf{U}$ have a common domain.

A binary relation $\succeq$ on $X$ is countably orderdense if there exists a countable $Y \subset X$ such that for all $x, z \in X$ with $x \succ z$ (i.e., $x \succeq z$ and not $z$ $\succeq x$ ), there exists a $y \in Y$ such that $x \succeq y \succeq z$.

THEOREM 1: If $\succeq$ is reflexive, transitive, and countably order-dense, there is a psychology that induces $\succeq$.

Proofs can be found in the Appendix. Reflexivity, transitivity, and countable order-density are standard assumptions that underlie the existence of a utility representation for preferences. Indeed, the only difference between Theorem 1 and the standard representation result is that Theorem 1 does not suppose $\succeq$ is complete. Theorem 1 thus makes do with weaker conditions than the Efe A. Ok (2002) result on representing an incomplete preference relation $\succeq$ with a (possibly infinite) set of utility functions. Juan Dubra et al. (2001) show that an expected utility representation of possibly incomplete preferences on lotteries also requires no extra assumptions beyond the standard.

We turn to the ordering of the strength of psychologies. To keep things simple, we henceforth require all psychologies to be complete. So every $u \in \mathbf{U}$ is a utility representation for $\succeq_{\mathbf{U}}$.
DEFINITION 1: A psychology $\mathbf{U}$ is no stronger than a psychology $\mathbf{V}$ if and only if $\mathbf{U} \supset \mathbf{V}$.

Since set inclusion is transitive, the "no stronger than" relation on psychologies is transitive as well. But when $X$ has more than one element, the "no stronger than" relation is incomplete (e.g., if $\mathbf{U}$ consists of all utilities that represent some preference relation $\succeq$ and $\mathbf{V}$ consists of all utilities that represent some $\succeq^{\prime} \neq \succeq$, then $\mathbf{U} \cap$ $\mathbf{V}=\varnothing$ ). We define a "weaker than" relation on psychologies as the asymmetric part of the "no stronger than" relation: $\mathbf{U}$ is weaker than $\mathbf{V}$ if and only if $\mathbf{U}$ is no stronger than $\mathbf{V}$ and it is not the case that $\mathbf{V}$ is no stronger than $\mathbf{U}$.

To illustrate, consider three psychologies: $\mathbf{U}$ consists of every utility representation of some preference relation $\succeq, \mathbf{V}$ consists of some representation $u$ of $\succeq$ and all increasing affine transformations of $u$, and $\mathbf{W}$ contains only the single function $u$. Then $\mathbf{U}$ is weaker than $\mathbf{V}$, and $\mathbf{V}$ is weaker than $\mathbf{W}$ (for the former conclusion we need to assume that $u$ has at least three distinct points in its range). As this example indicates, weaker psychologies make fewer assertions about the utility experiences of agents and should therefore be easier for agents to assemble. To put the matter in reverse, as psychologies become stronger or smaller, the utility experiences described become more and more limited. An agent with the psychology $\mathbf{U}$ makes only ordinal preference judgments, while an agent with the psychology $\mathbf{V}$ makes the same ordinal judgments as the $\mathbf{U}$ agent, but in addition makes cardinal comparisons of utility. That is, for any $(x, y, z, w)$ with $u(z) \neq u(w)$, the $\mathbf{V}$ agent experiences a specific ratio of utility differences

$$
\frac{u(x)-u(y)}{u(z)-u(w)}
$$

Given $(x, y, z, w)$, all $v \in \mathbf{V}$ maintain the same value for this ratio. Finally an agent with psychology $\mathbf{W}$ not only makes the ordinal judgments of the $\mathbf{U}$ agent and the cardinal comparisons of the $\mathbf{V}$ agent, but can in addition assign an exact utility number to every consumption experience. Singleton psychologies such as $\mathbf{W}$ are so implausibly strong that they have never been employed in economics or measurement theory. Appropriately, therefore, our ordering of psychologies places singleton psychologies at a polar extreme: a singleton 
psychology can never be weaker than any other psychology.

\section{Properties of Utility and Orderings of Properties}

We now use the ordering over psychologies to generate orderings over properties of utility functions. Formally, a property $P$ is simply a set of functions into the real line, where the domains of the functions can differ, and a utility function $u: A \rightarrow \mathbb{R}$ satisfies property $P$ if and only if $u \in P$. We mostly consider familiar properties that are routinely imposed on utility functions. For instance, one can define the property of continuity as the set of all continuous functions from arbitrary subsets of $\mathbb{R}^{n}$ into the real line.

DEFINITION 2: A psychology $\mathbf{U}$ maximally satisfies property $P$ if and only if for each $u \in$ $\mathbf{U}, u$ satisfies $P$, and there does not exist a psychology $\mathbf{V} \supsetneq \mathbf{U}$ such that each $v \in \mathbf{V}$ satisfies $P$.

In words, a psychology maximally satisfies $P$ if it is largest among psychologies whose constituent utility functions all satisfy $P$. So, for example, a $\mathbf{U}$ with domain $\mathbb{R}_{+}^{n}$ would maximally satisfy the property of continuity if and only if $\mathbf{U}$ consists of all functions $u$ : $\mathbb{R}_{+}^{n} \rightarrow \mathbb{R}$ that are continuous and that represent the same preference relation. The domain of a psychology may determine whether it maximally satisfies a property. For instance, if $X \subset \mathbb{R}_{+}^{n}$ is finite, any psychology consisting of all utility functions that represent some $\succeq$ on $X$ maximally satisfies continuity, but this is certainly not the case if, say, $X=\mathbb{R}_{+}^{n}$.

The earlier ordering of psychologies suggests the following ordering of properties.

\section{DEFINITION 3:}

(i) Property $P$ is no stronger than property $Q$, or $P \succeq_{N S} Q$, if and only if for all $\mathbf{U}$ that maximally satisfy $P$ and all $\mathbf{V}$ that maximally satisfy $Q, \mathbf{U} \cap \mathbf{V} \neq \varnothing$ implies $\mathbf{U} \supset \mathbf{V}$.

(ii) Property $P$ is weaker than property $Q$, or $P \succeq_{W} Q$, if and only if $P \succeq_{N S} Q$ and not $Q \succeq_{N S} P$.

In words, $P$ is no weaker than $Q$ if, whenever $P$ and $Q$ are comparable (some utility satisfies both simultaneously), the psychology maximally satisfying $P$ is no stronger than the psychology maximally satisfying $Q$. The relations $\succeq_{N S}$ and $\succeq_{W}$ need not be transitive or complete. Since our ordering of psychologies itself is not complete, the incompleteness is to be expected. Still, the incompleteness of $\succeq_{W}$ can be extensive since $P$ and $Q$ are unranked by $\succeq_{W}$ if $P$ and $Q$ are inconsistent, that is, if $P \cap Q=\varnothing$. For instance, let $P$ and $Q$ denote, respectively, the properties of strict concavity and strict convexity on nonsingleton convex subsets of $\mathbb{R}^{n}$. Then, since whenever a $\mathbf{U}$ maximally satisfies $P$ and a $\mathbf{V}$ maximally satisfies $Q$, it is always the case that $\mathbf{U} \cap \mathbf{V}=\varnothing$, we have both $P \succeq_{N S} Q$ and $Q \succeq_{N S} P$ and therefore neither $P \succeq_{W} Q$ nor $Q \succeq_{W} P$.

The intransitivity of $\succeq_{N S}$ and $\succeq_{W}$ may come as more of a surprise. We consider this subject in separate papers (Mandler, 2001, 2003) which show that there are natural domains on which $\succeq_{W}$, and related orderings are transitive or acyclic.

An alternative "weaker than" ordering will be useful later. First, we say a property $P$ intersects property $Q$ when $P \cap Q \neq \varnothing$.

DEFINITION 4: Property $P$ is strictly weaker than property $Q$, or $P \succeq_{S W} Q$, if and only if (1) $P$ intersects $Q$ and (2) whenever $\mathbf{U}$ maximally satisfies $P, \mathbf{V}$ maximally satisfies $Q$, and $\mathbf{U} \cap$ $\mathbf{V} \neq \varnothing$, then $\mathbf{U} \supsetneq \mathbf{V}$.

In contrast, property $P$ is weaker than $Q$ if it is merely the case that $P$ is no stronger than $Q$ and there is some $\mathbf{U}$ that maximally satisfies $P$ and some $\mathbf{V}$ that maximally satisfies $Q$ such that $\mathbf{U}$ is weaker (in the ordering of psychologies) than $\mathbf{V}$.

DEFINITION 5 (Ordinality): A psychology $\mathbf{U}$ is ordinal if and only if whenever $u \in \mathbf{U}$ then ( $v$ $\in \mathbf{U} \Leftrightarrow$ there exists an increasing transformation $g$ such that $v=g \circ u){ }^{2}$

If we say that two functions $u$ and vagree when, for all $x$ and $y$ in $X, u(x) \geq u(y) \Leftrightarrow v(x) \geq v(y)$, then a complete psychology $\mathbf{U}$ is ordinal if and

\footnotetext{
${ }^{2}$ A function $g: E \rightarrow \mathbb{R}$, where $E \subset \mathbb{R}$, is (a) an increasing transformation if and only if, when $x, y \in E$ and $x>y$, then $g(x)>g(y)$; and is (b) an increasing affine transformation if and only if there exist $a>0$ and $b$ such that, for all $x \in E$, $g(x)=a x+b$.
} 
only if $u \in \mathbf{U}$ implies that when $v$ agrees with $u$ then $v \in \mathbf{U}$.

DEFINITION 6 (Cardinality): A psychology $\mathbf{U}$ is cardinal if and only if whenever $u \in \mathbf{U}$ then $(v$ $\in \mathbf{U} \Leftrightarrow$ there exists an increasing affine transformation $g$ such that $v=g \circ u$ ).

Keep in mind that utility functions are the primitive. Consequently, theories that are sometimes called cardinal, such as the von NeumannMorgenstern theory of preferences over lotteries, do not necessarily qualify as cardinal in the current schema. For instance, let the domain be the set of lotteries over $n$ prizes, $X=\left\{p \in \mathbb{R}_{+}^{n}\right.$ : $\left.\sum_{i=1}^{n} p_{i}=1\right\}$. While it is true that an agent with vNM preferences $\succeq$ over $X$ will have as one of its utilities a function $v$ of the form $v(p)=\sum_{i=1}^{n}$ $p_{i} v_{i}$, the psychology of the agent is a distinct entity. The agent could have (a) an ordinal psychology consisting of all increasing transformations of $v$, (b) a cardinal psychology consisting of all increasing affine transformations of $v$, (c) some other cardinal psychology consisting of all increasing affine transformation of some utility that does not take the expected utility form, or (d) a psychology that is neither ordinal nor cardinal. It may be plausible that $\mathrm{vNM}$ agents will make cardinal comparisons of utility that are representable by their vNM utility functions, but the vNM axioms do not entail this conclusion.

We now define properties of utility as ordinal or cardinal and consider a couple examples.

DEFINITION 7: A property $P$ is ordinal (resp. cardinal) if and only if any $\mathbf{U}$ that maximally satisfies $P$ is ordinal (resp. cardinal).

To illustrate how these definitions work, consider the ordinal property of quasiconcavity. Define a function $u: Z \rightarrow \mathbb{R}$ to be quasiconcave if $Z$ is a convex set and, for all $x, y \in Z$ and $\lambda \in$ $[0,1], u(\lambda x+(1-\lambda) y) \geq \min \{u(x), u(y)\}$. To confirm that quasiconcavity is ordinal, let $\mathbf{U}$ maximally satisfy quasiconcavity and let $u$ be an arbitrary element of $\mathbf{U}$. Then, if $u$ and $v$ agree, there is an increasing transformation $f$ : Range $u \rightarrow \mathbb{R}$ such that $f \circ u=v$; since $f$ is increasing, for all $x, y \in Z$ and all $\lambda \in[0,1]$, $v(\lambda x+(1-\lambda) y) \geq \min \{v(x), v(y)\}$. Hence $v$ satisfies quasiconcavity and so, by Definition 2, $v \in \mathbf{U}$.
Additive separability will serve as a useful example of a cardinal property.

DEFINITION 8: A function $u: A \rightarrow \mathbb{R}$ satisfies additive separability if and only if, for some integer $n \geq 2$, there exist component spaces $A_{i}$, $i=1, \ldots, n$, such that $A=A_{1} \times \cdots \times A_{n}$ and functions $u_{i}: A_{i} \rightarrow \mathbb{R}, i=1, \ldots, n$, such that (1) for each $x \in A, u(x)=\sum_{i=1}^{n} u_{i}\left(x_{i}\right)$, (2) for each component $i$, Range $u_{i}$ is an interval, and (3) at least two of these intervals have nonempty interior.

The property of additive separability consists of the set of all functions that satisfy additive separability.

THEOREM 2: The property of additive separability is cardinal.

For a proof, see David H. Krantz et al. (1971), or for a more general cardinality result for additively separable functions, Mandler (2001). Theorem 2 provides the basis for Theorem 5, which establishes the cardinality of the ArrowKoopmans theory of convex preferences.

\section{Convexity of Preferences}

We consider two rationales for convexity and assess their measurement implications.

\section{A. Concavity as a Primitive}

The preferences $\succeq$ defined on the domain $X \subset \mathbb{R}^{n}$ are convex if and only if, for all $y \in X$, the set $\{x \in X: x \succeq y\}$ is convex. A function $u$ : $Z \rightarrow \mathbb{R}$ satisfies concavity if and only if $Z \subset \mathbb{R}^{n}$ is a convex set and, for all $x, y \in Z$ and all $\lambda \in$ $[0,1], u(\lambda x+(1-\lambda) y) \geq \lambda u(x)+(1-$ $\lambda) u(y)$. As is well known, if the preferences $\succeq$ have a utility representation that satisfies concavity, then $\succeq$ is convex. So, if a psychology $\mathbf{U}$ maximally satisfies concavity, then $\succeq_{\mathbf{U}}$ is convex.

Concavity, which means that agents experience diminishing marginal increments of satisfaction as they progressively increase their consumption along any line, has long served as an informal rationale for why preferences should be convex. But since assumptions on utility are usually labeled as either ordinal or cardinal, concavity has appeared to be cardinal, thus adding to concavity's illegitimacy. The 
next theorem shows, however, that concavity is in fact weaker than cardinality. Recall that property $P$ intersects property $Q$ if $P \cap Q \neq \varnothing$.

DEFINITION 9: Property $P$ has range $>n$ if and only if, for each $u \in P, \mid$ Range $u \mid>n$.

\section{THEOREM 3:}

(i) Any ordinal property is no stronger than concavity, and concavity is no stronger than any cardinal property.

(ii) Concavity is strictly weaker than any cardinal property with range $>2$ that intersects concavity.

(iii) Any ordinal property with range $>1$ that intersects the property of being concave and continuous is strictly weaker than the property of being concave and continuous.

Remark. Due to the fact that a concave function need not be continuous on the boundary of its domain, there are nontrivial preferenceseven preferences that exhibit strict preference between arbitrarily many pairs of consumption bundles - whose only utility representations are concave. It follows that there are ordinal properties with arbitrarily large range that are not weaker than concavity.

Theorem 3 (ii) reports an important fact about concavity. Along a line, concavity as a psychology assumes that an agent experiences each successive unit of consumption as delivering a smaller increment of utility or satisfaction. But concavity does not require that each utility increment is a specific fraction of the previous increment: agents experience diminishing marginal utility but no additional extra-ordinal precision. Cardinality, in contrast, requires that agents can make cardinal comparisons of utility. Hence, the ratio of utility increments for some given pair of consumption changes can assume only one value: a cardinal agent who satisfies concavity would be able to say that an additional unit of consumption delivers additional utility equal to some specific percentage of the previous unit of consumption. Cardinality thus requires an agent to make an implausibly extensive set of psychological judgments.

Concavity can be ranked relative to some other classical assumptions in utility theory. If $X$ is a convex nonempty open subset of $\mathbb{R}^{n}$, the property of continuity on $X$ is weaker than con- cavity on $X$, and the property of being continuous and nonconstant on $X$ is strictly weaker than concavity on $X$. If $Y \subset \mathbb{R}^{n}$ contains a nonempty open set, then any ordinal property that is nonconstant on $Y$ is strictly weaker than continuity on $Y$. These assertions follow from the fact that any concave function on an open set is continuous, but not vice versa, and the fact that any continuous increasing transformation preserves continuity, but noncontinuous increasing transformations do not preserve continuity. We omit the details, which vary only slightly from the proof of Theorem 3 .

The properties of continuity and concavitywith-continuity are each associated with a set of utility transformations, respectively increasing continuous and increasing concave functions from $\mathbb{R}$ to $\mathbb{R}$. This feature is by no means shared by all properties of utility. Moreover, even in these cases, the properties should not be confused with their associated transformations: the transformation must be applied to a function that satisfies the property in question. For instance, an increasing concave transformation of a nonconcave utility need not be concave.

\section{B. The Arrow-Koopmans Theory}

Arrow (1951), following unpublished remarks by Koopmans, argued that if an agent holds a consumption bundle for a period of time, say the interval $[0, T]$, and can decide on the timing of how that bundle is consumed, then the agent's preferences must be convex. W. M. Gorman (1957) later proposed a similar theory. Arrow implicitly supposed that the agent's total utility is the integral of the instantaneous utility achieved at each moment from 0 to $T$. An agent holding consumption bundle $x \in$ $\mathbb{R}_{+}^{n}$ chooses a function $\alpha$ from $[0, T]$ to $\mathbb{R}_{+}^{n}$ that maximizes this integral subject to the constraint that $\alpha$ integrates to no more than $x$. The informal argument for convexity is that an agent holding the bundle $\lambda x+(1-\lambda) y, \lambda \in[0,1]$, could consume $\lambda x$ over $\lambda T$ units of time and $(1-\lambda) y$ during the remaining $(1-\lambda) T$ time units. If the agent's utility at each instant is independent of the consumption at other instants, $\lambda x$ consumed over $\lambda T$ time units should deliver utility equal to $\lambda$ times the utility of $x$ consumed over $T$ time units, and similarly for $(1-\lambda) y$. So, if $x$ and $y$ are indifferent, consuming $\lambda x$ for $\lambda T$ units of time and $(1-\lambda) y$ for 
$(1-\lambda) T$ time units will leave the agent exactly as well off as with $x$ or $y$. But the agent may find a better temporal consumption pattern when endowed with $\lambda x+(1-\lambda) y$ and thus be better off than with $x$ or $y$. Hence indifference curves are convex. Arrow argued that this account of convexity, unlike the supposedly cardinalist stories that rely on diminishing marginal utility, is a purely ordinal explanation.

We follow Birgit Grodal's (1974) formalization of the Arrow-Koopmans theory. An agent has a utility function $V: \mathbb{R}_{+}^{n} \rightarrow \mathbb{R}$ that takes the form

$$
\begin{gathered}
V(x)=\sup _{\alpha \in \mathcal{L}} \int_{0}^{T} u(\alpha(t), t) d \mu(t) \\
\text { s.t. } \int_{0}^{T} \alpha_{i}(t) d \mu(t) \leq x_{i}, \quad i=1, \ldots, n,
\end{gathered}
$$

where $u: \mathbb{R}_{+}^{n} \times[0, T] \rightarrow \mathbb{R}, \mu$ is Lebesgue measure, $t \mapsto u(\alpha(t), t)$ is integrable, $x \in \mathbb{R}_{+}^{n}$, and $\mathcal{L}$ is the set of Lebesgue measurable functions $\alpha:[0, T] \rightarrow \mathbb{R}^{n}$ such that $\alpha_{i}(t) \geq 0$ for $i=$ $1, \ldots, n$ and a.e. $t \in[0, T]$. Let $\succeq_{A K}$ denote the preference relation on $\mathbb{R}_{+}^{n}$ that $V$ is a utility representation of.

THEOREM 4 (Grodal, 1974): The utility function $V$ is concave and therefore $\succeq_{A K}$ is convex.

We turn to the measurement requirements of the utility $\int_{0}^{T} u(\alpha(t), t) d \mu(t)$ in the maximization problem that defines $V$ but now seen as a function from $\mathcal{L}$ to $\mathbb{R}$.

DEFINITION 10: A function $U: \mathcal{L} \rightarrow \mathbb{R}$ satisfies utility integrability if and only if, for some positive integer $n,(i)$ there exists a $u: \mathbb{R}_{+}^{n} \times[0$, $T] \rightarrow \mathbb{R}$ such that $t \mapsto u(\alpha(t), t)$ is integrable and $U(\alpha)=\int_{0}^{T} u(\alpha(t), t) d \mu(t)$ for all $\alpha \in \mathcal{L} ;(i i)$ $\mid$ Range $U \mid>1$.

Condition (ii) implies that Range $U$ is a nontrivial interval (see the proof of Theorem 5).

For a psychology $\mathbf{U}$ to maximally satisfy utility integrability, $\mathbf{U}$ must be the largest psychology such that each function $U \in \mathbf{U}$ satisfies utility integrability (cf. Definition 2). For the property of utility integrability to qualify as cardinal, every psychology $\mathbf{U}$ that maximally satisfies utility integrability must consist of, and only of, those functions that are the affine transformations of any function in $\mathbf{U}$.

THEOREM 5: Utility integrability is a cardinal property.

Theorems 5 and 3 together imply that the Arrow-Koopmans theory imposes stricter measurement requirements on agents than does concavity. Utility integrability has range $>2$ and intersects concavity; so Theorem 3 implies that concavity is strictly weaker than utility integrability. Thus, concavity, despite its preeminent place in preordinal utility theory, is nearer to ordinalist standards of measurability. These conclusions address only the measurement status of utility integrability; the Arrow-Koopmans theory is ingenious and despite its cardinality offers an intriguing explanation of why preferences should be convex.

\section{Concave Utilitarianism: Between Cardinal and Ordinal Interpersonal Comparisons}

It is common to think that even if positive preference theory should be based on purely ordinal assumptions, utilitarianism is necessarily a cardinal enterprise. But we will see that the utilitarian recommendations that command the broadest consensus rely on a measurement scale that is weaker than cardinality: concave psychologies reproduce the key utilitarian recommendation that income be redistributed from high-utility agents with low marginal utilities of income to low-utility agents with high marginal utilities of income. Not every utilitarian conclusion can be derived from concavity, but the missing results are the anti-egalitarian policies that harm low-utility agents. Even many utilitarians have viewed such recommendations as ethically suspect.

The formal theory of social choice concentrates on the standard measurement classes: ordinal scales, interval or cardinal scales, and occasionally ratio scales. Opening up the terrain between ordinal and cardinal measurement brings out new possibilities. Any version of utilitarianism based on a compromise measurement standard, such as the concavity proposal we lay out, has the advantage that it is easier to construct (requires fewer interpersonal judgments) than the traditional cardinal doctrine. 
Consider a society of $I$ agents who must choose from a set of social choices $X$. To keep a concrete example in mind, one we discuss later, $X \subset \mathbb{R}_{+}^{I}$ might be a set of feasible income profiles for the $I$ agents.

Interpersonal welfare comparisons will be represented by social utility functions $u$, which are standard utility functions except that they are defined on $\square \times X$, where $\llbracket$ denotes the set of agents $\{1, \ldots, I\}$. We interpret $u(i, x) \geq u(j, y)$ to mean that agent $i$ with social choice $x$ is at least as well off as agent $j$ with social choice $y$. So, in particular, $u(i, \bullet)$ gauges agent $i$ 's well-being. When combined with an aggregation rule, e.g., $\sum_{i \in \llbracket} u(i, x)$ or $\min _{i \in \rrbracket}$ $u(i, x)$, a social utility $u$ generates an ordering of $X$.

To assess the measurement implications of different ways of making interpersonal welfare comparisons, we again consider psychologies of utility functions. To keep the distinction between social and individual choice in mind, call a psychology $\mathbf{U}$ of social utility functions a social psychology. One may think of the interpersonal judgments represented by a social psychology as arising from the preferences of an individual contemplating what it would be like to be various agents under various social outcomes. A social psychology is then interpreted in the same way as an individual psychology: the psychology lists the utility functions that accurately compare various consumption experiences. It just so happens that for social psychologies the set of possible consumption experiences is $\square \times X$ rather than an arbitrary set.

As before, we consider only social psychologies $\mathbf{U}$ that are complete: $\mathbf{U}$ will contain only, though not necessarily all, increasing transformations of some $u \in \mathbf{U}$. In the language of social choice theory, completeness implies that the minimum measurement standard that any resulting social welfare function satisfies is ordinal level comparability (in the terminology of Kevin Roberts, 1980b). Since we do not require $\mathbf{U}$ to contain all ordinally equivalent social utilities, our social psychologies can obey a stronger measurement standard. ${ }^{3}$

\footnotetext{
${ }^{3}$ Some social choice theories consider utility transformations that vary as a function of the agent $i$, e.g., invariance with respects to individual origins of utility, used by Claude d'Aspremont and Louis Gevers (1977) to axiomatize utilitarianism, which admits affine transformations of the form $a u(i, \cdot)+b_{i}$. In our framework, these transformations translate into incomplete social psychologies.
}

We again compare cardinal, ordinal, and concave psychologies. Definitions 5 and 6 of ordinality and cardinality apply unchanged to social psychologies; we now use $\mathbf{U}_{O}$ and $\mathbf{U}_{C}$ to denote, respectively, generic ordinal and cardinal social psychologies. But we must redefine concavity, since, due to $\mathbb{\square}$, the domain of a social utility is not a convex set. We call a social utility function $u$ coordinately concave if and only if $X$ is convex and, for each $i \in \mathbb{}, u(i, \cdot)$ is concave and continuous. The property of coordinate concavity is then simply the set of all coordinately concave functions, and a concave social psychology is therefore a social psychology that maximally satisfies coordinate concavity. It is thus a largest social psychology that preserves the concavity of each agent's utility and where each social utility represents the same complete ordering on $\square \times$ $X$. $\mathbf{U}_{C C}$ will denote a generic concave social psychology.

We consider social welfare rankings that order the social choices $X$ according to the sum of the utilities $\sum_{i \in \llbracket} u(i, x)$. Given a social psychology $\mathbf{U}$, the ordering $\succeq_{\mathbf{U}}^{s}$ over $X$ is defined by

$x \succeq_{\mathbf{U}}^{s} y \Leftrightarrow \sum_{i \in \mathbb{\Omega}} v(i, x) \geq \sum_{i \in \mathbb{U}} v(i, y)$ for all $v \in \mathbf{U}$.

So $x$ is ranked above $y$ when all the social utilities in $\mathbf{U}$ rank $x$ above $y$ by a sum-ofutilities test. The superscript $s$ distinguishes $\succeq_{\mathbf{U}}^{s}$, an ordering over the social choices, from $\succeq_{\mathbf{U}}$, the ordering over $\llbracket \times X$ that any $u \in \mathbf{U}$ represents. While $\succeq_{\mathbf{U}}$ is complete, $\succeq_{\mathbf{U}}^{s}$ need not be: $\mathbf{U}$ might not specify enough social welfare judgments for utilitarian aggregation always to be decisive.

Different social psychologies evidently generate different "utilitarian" rankings. Call $\succeq_{\mathbf{U}_{C}}^{s}$, $\succeq_{\mathbf{U}_{o}}^{s}$, and $\succeq_{\mathbf{U}_{C C}}^{s}$ cardinal, ordinal, and concave utilitarianism, respectively. Cardinal utilitarianism is the usual utilitarianism; any $u$ in $\mathbf{U}_{C}$ generates the same sum-of-utilities ordering of social choices. But cardinal utilitarianism rests on a demanding measurement standard; specifying a cardinal social psychology requires making cardinal comparisons of utility across agents. Ordinal utilitarianism, at the other extreme, relies on a much weaker measurement standard. Since ordinal utilitarianism requires any ranking of $x$ over $y$ to pass a larger set of sum-of-utilities tests, it ranks fewer social 
choices than cardinal utilitarianism. Indeed, we will see that ordinal utilitarianism incompleteness can be so extensive that it makes trivially few rankings. Since a concave social psychology $\mathbf{U}_{C C}$ contains the utilities generated by the affine transformations of any $u \in \mathbf{U}_{C C}$, and since the set of all ordinal representations of $u$ contains any concave representation, concave utilitarianism produces more rankings than ordinal utilitarianism, but fewer rankings than cardinal utilitarianism. We write this down as a theorem.

THEOREM 6: Let the social psychologies $\mathbf{U}_{C}$ be cardinal, $\mathbf{U}_{O}$ be ordinal, and $\mathbf{U}_{C C}$ be coordinately concave. If $\mathbf{U}_{C} \cap \mathbf{U}_{C C} \neq \varnothing$, then $\mathbf{U}_{C} \subset$ $\mathbf{U}_{C C}$ and $\succeq_{\mathbf{U}_{C}}^{s} \supset \succeq_{\mathbf{U}_{C C}}^{s}$. If $\mathbf{U}_{C C} \cap \mathbf{U}_{O} \neq \varnothing$, then $\mathbf{U}_{C C} \subset \mathbf{U}_{O}$ and $\succeq_{\mathbf{U}_{C C}}^{s} \supset \succeq_{\mathbf{U}_{O}}^{s}$.

Concave utilitarianism relaxes the stringent measurement requirements of cardinal utilitarianism, and, as in the case of preference theory, concavity as a psychology pinpoints the characteristically neoclassical views of economic psychology. But does concave utilitarianism retain the egalitarianism and enough of cardinal utilitarianism's decisiveness? Specifically, does concave utilitarianism recommend redistributions from rich to poor and does it rank sufficiently many social choices?

An ordering $\succeq_{\mathbf{U}}^{s}$ and a particular $u$ in $\mathbf{U}$ induce an ordering over utility vectors in $\mathbb{R}^{I}$ that will help characterize $\succeq_{\mathbf{U}}^{s}$. Given a social psychology $\mathbf{U}$ and $u \in \mathbf{U}$, we define $R_{u \in \mathbf{U}}$, an ordering over vectors in $\mathbb{R}^{I}$ (distinguished subsequently by letters in bold) as follows.

DEFINITION 11: Given a social psychology $\mathbf{U}, \mathbf{v} R_{u \in \mathbf{U}} \mathbf{w} \Leftrightarrow$ there exist $x, y \in X$ with $\mathbf{v}=$ $(u(1, x), \ldots, u(I, x)), \mathbf{w}=(u(1, y), \ldots, u(I, y))$ and $x \geq_{\mathbf{U}}^{s} y$.

We say that $\mathbf{u}$ is feasible for a social utility $u$ if and only if

$$
\begin{aligned}
& \mathbf{u} \in F(u) \equiv\left\{\mathbf{u} \in \mathbb{R}^{I}: \text { there exists } x \in X\right. \\
& \text { such that } \mathbf{u}=(u(1, x), \ldots, u(I, x))\} .
\end{aligned}
$$

Not surprisingly, $\mathbf{v}$ is ranked higher than $\mathbf{w}$ according to any cardinal utilitarian ordering $R_{u \in \mathbf{U}_{C}}$ if and only if the sum of the coordinates in $\mathbf{v}$ is at least as large as the sum in $\mathbf{w}$. Ordinal utilitarianism is also easily characterized. For any vector $\mathbf{v}$, let $\mathbf{v}^{*}$ denote the vector formed by placing the coordinates $\mathbf{v}_{1}, \ldots, \mathbf{v}_{I}$ in increasing order.

\section{THEOREM 7:}

(i) If $\mathbf{U}_{C}$ is cardinal, $u \in \mathbf{U}_{C}$, and $\mathbf{v}, \mathbf{w}$ are feasible for $u$, then $\mathbf{v} R_{u \in \mathbf{U}_{C}} \mathbf{w} \Leftrightarrow \sum_{i \in \mathbb{}} \mathbf{v}_{i} \geq$ $\sum_{i \in \llbracket} \mathbf{w}_{i}$.

(ii) If $\mathbf{U}_{O}$ is ordinal, $u \in \mathbf{U}_{O}$, and $\mathbf{v}, \mathbf{w}$ are feasible for $u$, then $\mathbf{v} R_{u \in \mathbf{U}_{o}} \mathbf{w} \Leftrightarrow \mathbf{v}^{*} \geq \mathbf{w}^{*}$.

Result (ii) says that ordinal utilitarianism judges $x \succeq_{\mathbf{U}_{O}}^{s} y$ if and only if, according to any $u \in \mathbf{U}_{O}$, the $i$ th best-off agent under $x$ is at least as well off as the $i$ th best off under $y$ for all $i \in$ 『. Thus, modulo utilitarianism's anonymity requirement (which implies that if $\mathbf{u}$ and $\mathbf{v}$ merely rearrange indices without changing the utility level of the $i$ th best-off agent for any $i$, then $\mathbf{u}$ and $\mathbf{v}$ are tied according to $R_{u \in \mathbf{U}_{o}}$ ), ordinal utilitarianism recommends only Pareto improvements. We will say that $\mathbf{u}$ is an anonymous Pareto improvement over $\mathbf{v}$ if $\mathbf{u}^{*} \geq \mathbf{v}^{*}$ (sometimes this is called a Suppes-Sen improvement). Ordinal utilitarianism lacks the traditional redistributive conclusions of utilitarianism: it will never recommend a small transfer of wealth from a high-utility agent to a low-utility agent. Cardinal utilitarianism of course recommends such transfers if the low-utility agent has a higher marginal utility of wealth (according to any given $u \in \mathbf{U}_{C}$ ) than the high-utility agent.

To characterize concave utilitarianism, first recall the definition of a least concave function. Let $\mathbf{V}$ denote an individual psychology consisting of all the continuous and concave utility functions, defined on some convex domain $X \subset$ $\mathbb{R}^{\mathrm{n}}$, that represent some fixed preference relation $\succeq$ on $X$. The function $\bar{v} \in \mathbf{V}$ is least concave if and only if for every $v \in \mathbf{V}$ there exists an increasing concave transformation $g$ : Range $\bar{v} \rightarrow \mathbb{R}$ such that $v=g \circ \bar{v}$. Gerard Debreu (1976) proved that a least concave $\bar{v}$ exists. Social psychologies do not have convex domains, however, and so the definition of least concavity needs amendment. Let $\bar{u}(i, X)$ denote the range of the function $\bar{u}(i, \cdot)$.

DEFINITION 12: If $\mathbf{U}_{C C}$ is a concave social psychology, then $\bar{u} \in \mathbf{U}_{C C}$ is least coordinately concave or lcc if and only if for all $u \in \mathbf{U}_{C C}$ 


\begin{tabular}{|l|l|l|l|l|l|l|}
\hline tapraid1/z3y-aer/z3y-aer/z3y00406/z3y1807d06a & longd & S=31 & 8/7/06 & 14:17 & Art: 20030804 & Input-dld(dld) \\
\hline
\end{tabular}

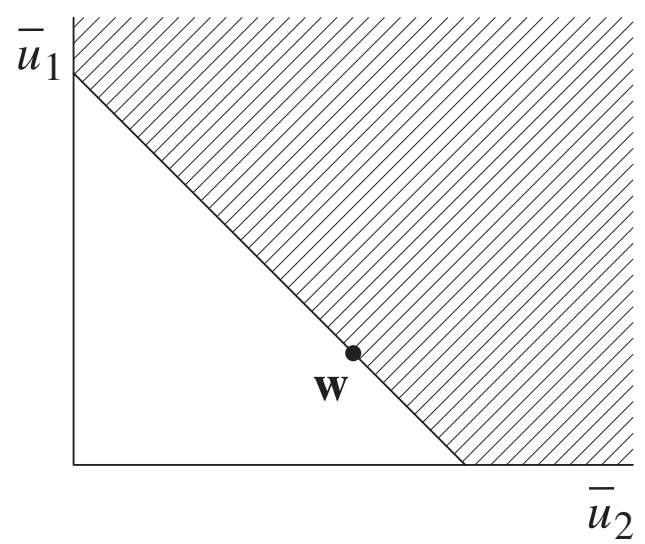

Figure 1. Cardinal Utilitarian Upper Contour Set

there exists an increasing transformation $g$ : Range $\bar{u} \rightarrow \mathbb{R}$ such that $u=g \circ \bar{u}$ and, for all $i \in \mathbb{Q}, g$ is concave when restricted to $\bar{u}(i, X)$.

Debreu's least concavity theorem does not itself imply that each $\mathbf{U}_{C C}$ contains an lcc utility function, but the proof extends easily. Let con $S$ denote the convex hull of a set $S$.

THEOREM 8: If $\mathbf{U}_{C C}$ is coordinately concave, $\bar{u} \in \mathbf{U}_{C C}$ is lcc, and $\mathbf{v}, \mathbf{w}$ are feasible for $\bar{u}$, then

$$
\begin{gathered}
\mathbf{v} \in \operatorname{con}\left\{\mathbf{u} \in F(\bar{u}): \mathbf{u}^{*} \geq \mathbf{w}^{*}\right\} \\
\Rightarrow \mathbf{v} R_{\bar{u} \in \mathbf{U}_{C C}} \mathbf{w}
\end{gathered}
$$

So if $(\bar{u}(1, x), \ldots, \bar{u}(I, x))^{*}$ is a convex combination of Pareto improvements of $(\bar{u}(1, y), \ldots$, $\bar{u}(I, y))^{*}$ then $x \succeq_{\mathbf{U}_{C C}}^{s} y$.

The upper contour sets of the three utilitarianisms clarify what Theorem 8 says. Suppose $I=2$ and that $\mathbf{U}_{C}, \mathbf{U}_{O}, \mathbf{U}_{C C}$ have a common element $\bar{u}$ that is lcc for $\mathbf{U}_{C C}$. To keep things simple, suppose $F(\bar{u})$ equals $\mathbb{R}_{+}^{I}$. Fixing an arbitrary $\mathbf{w}$, the upper contour sets $\{\mathbf{v}: \mathbf{v} R \mathbf{w}\}$ for the three utilitarian orderings $R \in\left\{R_{\bar{u} \in \mathbf{U}_{\bar{C}}}\right.$, $\left.R_{\bar{u} \in \mathbf{U}_{o}}, R_{\bar{u} \in \mathbf{U}_{C C}}\right\}$ are pictured in Figures 1 F1-3 through 3. In this two-dimensional case, $\{\mathbf{v}$ : $\left.\mathbf{v} R_{\bar{u} \in \mathbf{U}_{C C}} \mathbf{w}\right\}$ exactly coincides with the convex hull given in Theorem 8 (see Theorem 9 below).

Figures 2 and 3 show that concave utilitarianism ranks a much richer set of utility vectors than ordinal utilitarianism. Not only are the

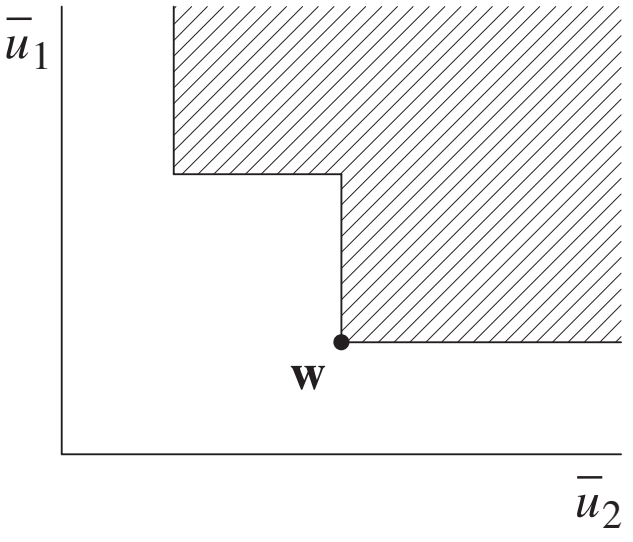

Figure 2. Ordinal Utilitarian UpPer Contour Set

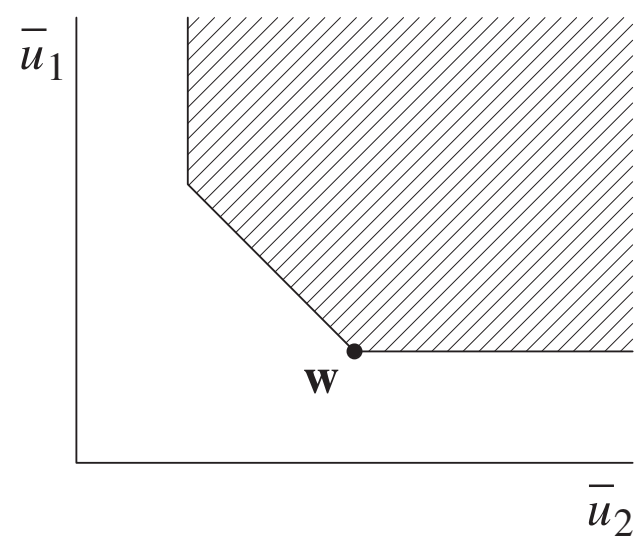

Figure 3. Concave Utilitarian Upper Contour Set

anonymous Pareto improvements weakly superior to $\mathbf{w}$, but any policy that benefits a utilitypoor agent at the expense of a utility-rich agent with no net loss of least concave utility is also superior. On the other hand, in contrast to cardinal utilitarianism, concave utilitarianism does not declare any utility vector that lowers the welfare of the worse-off agent to be superior to w. Indeed, for pairs of agents, changes from $\mathbf{w}$ that harm the worse-off agent are the only orderings made by $R_{\bar{u} \in \mathbf{U}_{C}}$ but not by $R_{\bar{u} \in \mathbf{U}_{C F}}$. Concave utilitarianism thus stakes out an egalitarian compromise between standard cardinal utilitarianism and the narrow Paretian judgments made by ordinal utilitarianism. 
Cardinal utilitarianism has long been criticized for ignoring welfare levels and in particular for recommending that low-utility agents should undergo arbitrarily large utility losses whenever those losses lead to greater utility gains for high-utility agents. Concave utilitarianism does not suffer from this defect. Moreover, concave utilitarianism does not arrive at its prohibition on harming the least well-off by invoking an equity axiom (as in Hammond, 1976). Rather, the egalitarianism stems directly from the psychological content of concavity.

We can characterize this egalitarianism by turning to the second-order stochastic dominance and poverty ordering literatures (see G. Hanoch and Haim Levy, 1969; Serge-Christophe Kolm, 1969; Vijay S. Bawa, 1975; Charles Blackorby and David Donaldson, 1977; Anthony F. Shorrocks, 1983; James E. Foster and Shorrocks, 1998a, 1988b; and the discussion of Roberts, 1980b, below). In the literature on poverty orderings, more equal distributions of income will, $\mathrm{ce}$ teris paribus, raise the sum of utilities, no matter what concave function maps individual income to social welfare. In the current setting, the utility levels given by the least coordinately concave social utility functions play the role of incomes, and thus more equal distributions of least concave utility with no net loss will be a concave-utilitarian improvement, no matter how concave individual utility functions are. Thus, even when $X$ specifies consumption of many commodities, agents' welfare can be compared using a single-dimensional variable-least concave utility - analogous to income. In effect then, we give a measurement theory and multicommodity rationale for the ordering of utility distributions or income distributions cited above.

Our characterization relies on the historically important Pigou-Dalton principle (see, e.g., Hervé Moulin, 1988).

Given a social utility $u$, an ordering $R$ over utility vectors satisfies the Pigou-Dalton principle if, for any $j, k \in \mathbb{\square}$ and any $\mathbf{v}, \mathbf{w}$ feasible for $u$ with $\mathbf{v}_{m}=\mathbf{w}_{m}$ for $m \notin\{j, k\}$ :

$$
\mathbf{v}_{j}+\mathbf{v}_{k}=\mathbf{w}_{j}+\mathbf{w}_{k} \text { and } \min \left\{\mathbf{v}_{j}, \mathbf{v}_{k}\right\} \geq \min \left\{\mathbf{w}_{j}, \mathbf{w}_{k}\right\}
$$

$$
\Rightarrow \mathbf{v} R \mathbf{w} \text {. }
$$

In words, $R$ satisfies Pigou-Dalton if a switch to a new vector of utilities that affects only a pair of agents is recommended when the sum of utilities is unchanged, and the lower-utility agent after the switch is no worse off than the lower-utility agent before the switch.

Figure 3 suggests that concave utilitarianism should satisfy the Pigou-Dalton principle. Subject to one caveat, the implication in (PD) in fact goes both ways if we replace the "=" in (PD) with " $\geq$," as Theorem 9 reports. In this sense, Pigou-Dalton characterizes concave utilitarianism. The caveat arises when a social utility function assigns ranges for the individual utility functions that do not overlap. For example, suppose two agents $a$ and $b$ must split a pie and some concave social utility function gives $a$ and $b$ sets of achievable utilities that are disjoint. One may then multiply either $a$ 's or $b$ 's utility by any positive constant and thereby arrive at another concave social utility. So a concave social psychology could not judge either agent's marginal utility of consumption to be higher or lower than the other's, thereby emptying concave utilitarianism of its egalitarian content. To exclude this wrinkle, it is sufficient to assume for any $u \in \mathbf{U}_{C C}$ that $\cup_{i \in \llbracket} \operatorname{Int} u(i, X)$ is an interval (where 'Int' denotes interior). The range of each agent's utility then does directly or indirectly overlap the range of any other agent's utility; arbitrary rescalings of a single individual's utility function are therefore blocked, and nontrivial interpersonal comparisons can be made.

THEOREM 9: Let $\bar{u}$ be lcc for $\mathbf{U}_{C C}$ and suppose $\cup_{i \in \llbracket}$ Int $\bar{u}(i, X)$ is an interval. If $\mathbf{v}, \mathbf{w}$ are feasible for $\bar{u}$ and identical in all but two coordinates $j$ and $k$, then

$$
\begin{gathered}
\mathbf{v}_{j}+\mathbf{v}_{k} \geq \mathbf{w}_{j}+\mathbf{w}_{k} \text { and } \min \left\{\mathbf{v}_{j}, \mathbf{v}_{k}\right\} \geq \min \left\{\mathbf{w}_{j}, \mathbf{w}_{k}\right\} \\
\Leftrightarrow \mathbf{v} R_{\bar{u} \in \mathbf{U}_{C C}} \mathbf{w} .
\end{gathered}
$$

Thus we arrive at a "poverty ordering" of utility distributions despite beginning with preferences defined over an $X$ that can specify consumption of an arbitrary number of goods.

A contrasting result of Avinash K. Dixit and Jesus Seade (1979) pins down the content of Theorem 9. Dixit and Seade show in effect that there are preferences $\succeq$ on $\mathbb{R}^{n}$ and $x \in \mathbb{R}^{n}$, with $\succeq$ representable by a smooth increasing concave utility function, such that for any such 
representation $u$ there is a $y$ near $x$ with $u(y)>u(x)$ and $D u(y) \gg D u(x)$. If there were two agents, each with the preferences $\succeq$, but one endowed with $x$ and the other endowed with $y$, then a planner who assigned $u$ to both agents and ranked allocations according to the sum of utilities would always recommend at least some transfer of goods from the agent with the low utility level (the " $x$ agent") to the agent with the high utility level (the " $y$ agent"). Theorem 9 does not contradict this result. Dixit and Seade let $y$ vary as a function of the concave representation $u$ : given $u$, there exists a utility-rich $y$ agent to whom a transfer from the $x$-agent would raise the sum of the $u$ 's. A concave utilitarian in contrast would require any transfer to raise the sum of utilities calculated with all concave $u$ 's, and any transfer from some specific $y$ agent to the $x$ agent would be rejected by a sufficiently concave $u$.

To illustrate cardinal and concave utilitarianism's common ground, and the paucity of ordinal utilitarian rankings, consider the canonical problem of ranking distributions of a single good.

Example.-A policymaker must choose a distribution of the aggregate output $\omega>0$, and so the set of social choices $X$ is $\left\{x \in \mathbb{R}_{+}^{I}: \Sigma_{i \in \mathbb{0}}\right.$ $\left.x_{i}=\omega\right\}$. We assume that the social psychologies $\mathbf{U}_{C}, \mathbf{U}_{O}$, and $\mathbf{U}_{C C}$ have a common $u$ such that each $u(i, \cdot)$ is increasing, differentiable, and strictly concave in $x_{i}$ (the $i$ th coordinate of $x, i$ 's income) and constant in the other coordinates. Let $u(i, \cdot)$ henceforth denote social utility as a function of $x_{i}$ alone. Suppose $\bar{u}$ is lcc for $\mathbf{U}_{C C}$ and that $\cup_{i \in \mathbb{}}$ Int $\bar{u}(i, X)$ is an interval.

First, let all individuals be identical: $u(i, \cdot)=$ $u(j, \cdot)$ for any $i, j \in \mathbb{l}$. Although the cardinal and concave utilitarian orderings $\succeq_{\mathbf{U}_{C}}^{s}$ and $\succeq_{\mathbf{U}_{C C}}^{s}$ are not identical, they both rank $e=((1 / I) \omega, \ldots$, $(1 / I) \omega)$ above any other distribution in $X$. For $\succeq_{\mathbf{U}_{o}}^{s}$, in contrast, any $x \in X$ that varies by agent is unranked relative to $e$, and no pair of points in $X$ is strictly ranked.

Next, allow $u(i, \cdot) \neq u(j, \cdot)$ and let $D u\left(k, x_{k}\right)$ denote the derivative of $u(k, \cdot)$, evaluated at $x_{k}$. As in the identical-agent case, $\succeq_{\mathbf{U}_{C}}^{s}$ ranks an $x$ such that $D u\left(i, x_{i}\right)=D u\left(j, x_{j}\right)$ to be superior to any other point in $X$. While $\succeq_{\mathbf{U}_{C C}}^{s}$ no longer has to make this ranking, Theorem 9 implies, given some base distribution $x$, that some transfer of income from agent $j$ to agent $i$ will be superior according to $\succeq_{\mathbf{U}_{C C}^{s}}^{s}$ if and only if

$$
\begin{aligned}
& D \bar{u}\left(i, x_{i}\right)>D \bar{u}\left(j, x_{j}\right) \\
& \text { and } \bar{u}\left(i, x_{i}\right)<\bar{u}\left(j, x_{j}\right) .
\end{aligned}
$$

So, rather than the equal derivative condition, any $x$ such that

$$
\left(D \bar{u}\left(i, x_{i}\right)-D \bar{u}\left(j, x_{j}\right)\right)\left(\bar{u}\left(i, x_{i}\right)-\bar{u}\left(j, x_{j}\right)\right) \geq 0
$$

for all $(i, j)$ is undominated according to $\succeq_{\mathbf{U}_{C C}}^{s}$ : agents' utility levels and their marginal utilities of income (both calculated using $\bar{u}$ ) must be comonotonic. Ordinal utilitarianism again makes very few orderings: given some $x \in X$, no transfer from a lower utility agent to a higher utility agent is ranked relative to $x$, nor is any small transfer from a higher utility agent to a lower utility agent. And any point in $X$ that leads to equal utility levels is always unranked relative to all other points in $X$.

Notice how easy it is in this example for a concave utilitarian to make policy decisions. A policymaker does not have to posit exact ratios of different agents' gains in utility. To judge a small transfer, the policymaker need only decide if a transfer from $i$ to $j$ leads to a bigger gain for $j$ than $i$ 's loss and if $i$ 's ex ante welfare level is higher than $j$ 's.

Although the cardinal and concave utilitarian orderings are not the same, it is concave rather than cardinal utilitarianism that provides the better rationale for the positions endorsed by the original neoclassical utilitarians. The connection to the Pigou-Dalton principle is revealing. Arthur C. Pigou, the architect of neoclassical welfare economics, considered himself both a cardinalist and a utilitarian: at least in theory, each individual in society has a cardinal and interpersonally comparable utility function, and policies should be evaluated by summing the utility numbers that they lead to. But Pigou (1932) recognized that it is not easy to come by cardinal utility information, and therefore argued that, as a practical matter, welfare economics can validate only policies that do not rely on that information. Even 
Edgeworth (1897), the arch-utilitarian, did not approve of policies whose justification depends on the specific choice of a cardinal utility function rather than on DMU alone (Mandler, 1999, chap. 6). In Pigou's view, a policy that lowers the income of a low-utility individual by $\$ 1$ and raises the income of a high-utility individual by $\$ x$ cannot be backed unambiguously, no matter how large $x$ is, since the marginal utility of income might be very small at high utility levels. On the other hand, if the low-utility agent gains by $\$ y, y>$ 1 , and the high-utility agent loses by $\$ 1$, then any sum-of-utilities test will recommend the policy change if agents share a concave utility of income. Concave utilitarianism backs the same recommendations, but it elevates the theoretical status of not having cardinal information about individuals. According to concavity as a social psychology, there is no unknown but nevertheless real cardinal utility function lurking out there-the most detailed information that could in principle be known about interpersonally comparable utility is that it is concave. The close match between the theory of concave utilitarianism and $\mathrm{Pi}$ gou's practical position is unsurprising: concave utilitarianism imposes exactly the psychological content that informed Pigou's account of human happiness.

Finally, we can characterize a concaveutilitarian comparison of utility vectors that have arbitrarily many coordinate changes by:

$$
\mathbf{v} R_{\bar{u} \in \mathbf{U}_{C C}} \mathbf{w} \Leftrightarrow \sum_{i=1}^{n} \mathbf{v}_{i}^{*} \geq \sum_{i=1}^{n} \mathbf{w}_{i}^{*} \text { for all } n \in \mathbb{U},
$$

where $\mathbf{v}, \mathbf{w} \in F(\bar{u})$ and where we again assume the same range condition that $\cup_{i \in \rrbracket}$ Int $\bar{u}(i, X)$ is an interval obtains. Kevin Roberts (1980b) essentially hypothesized this equivalence (in different terminology) and suggested Hanoch and Levy (1969) and least concave utilities as the appropriate tools. Roberts did not recognize that social utilities cannot satisfy the traditional definition of least concavity, an omission that obscured the need for the range condition. We should point out that Roberts did not take sets of concave utility functions as primitive, but argued, like Pigou, that such a set can be assumed to contain the one "true" cardinal family of utilities.

\section{Observable Tests of Concavity}

Cardinal utility theory was rejected not because it invoked mental relationships above and beyond choice behavior; psychology routinely does just this without tarnishing its scientific standing. Cardinality ran into trouble because, first, there was no convincing evidence that satisfaction is a cardinal quantity and, second, cardinal utility seemingly had no role to play in consumer theory. Although these criticisms are telling, they do not apply to all forms of nonordinal utility. With regard to the second charge, we have seen that concave psychologies do serve an important purpose even in positive theory-they provide a concise and only mildly nonordinal rationale for the convexity of preferences.

As for the first charge, the MRI revolution in cognitive neuroscience has identified neural correlates of consumption decisions that can test whether an agent has a concave psychology. As we will see, a concavity test requires only that neural correlates have two properties: they should map into an agent's ordinal preference ranking of alternatives and they should order the gains or losses in well-being that an agent experiences relative to well-being at the agent's reference consumption level. Such evidence cannot always be used to test for cardinality, which can require more extensive data.

Neuroscientists have uncovered various indicators of choice decisions. Brian Knutson et al. (2001), Martin P. Paulus et al. (2001), and Hans C. Breiter et al. (2001) show the extent of activity in several brain areas (the nucleus accumbens, the parietal cortex, and the sublenticular extended amygdala, among others) that correlate with the magnitude of monetary rewards that subjects receive. ${ }^{4}$ Agents' money gains come on top of their base or reference consumption levels: background income and consumption are fixed and the experiments establish benchmark levels of neural response. Also, the correlates of economic gains turn out to occur largely at one set of neural sites, while correlates of losses occur at others (Breiter et al., 2001). While this fact is consistent with subjects

\footnotetext{
${ }^{4}$ The recent special issue on neuroeconomics in Games and Economic Behavior (Aldo Rustichini, 2005) gives an overview of the neuroeconomics literature. See also Paul W. Glimcher and Rustichini (2004).
} 
having stable ordinal preferences, it underscores that neural indicators give evidence of changes relative to reference consumption. If current progress continues, this literature may well uncover neural indicators that reliably track the consumption increments that agents prefer and choose.

We suppose therefore that an agent who first consumes a base or reference consumption bundle $c$ and then adds an increment $y$ to $c$ displays an identifiable neural response, which we label $N(c, y)$. (It may help to think of $c$ and $c+y$ as flows over a brief period.) Each $N(c, y)$ may well lie on a unidimensional or multidimensional scale, but the range of $N$ could also consist of qualitative events with no numerical structure. We suppose the agent has a strict preference relation $\succ$ defined over consumption vectors that is a weak order (asymmetric and negatively transitive). The relation $\succ$ will remain the same for all base bundles $c$ : for any $c$ the agent always chooses $y$ over $y^{\prime}$ when $c+$ $y \succ c+y^{\prime}$. As usual, $\succ$ is confirmable from choice data. The assumption that $N$ correlates with preference means that (a) strictly preferred bundles lead to distinct neural observations and (b) whenever the same pair of observations arises, the same direction of preference obtains. Formally these properties for $N$ mean that for all reference bundles $c, c^{\prime}$, and increments $y, y^{\prime}, z, z^{\prime}$ :

$$
c+y \succ c+y^{\prime} \Rightarrow N(c, y) \neq N\left(c, y^{\prime}\right),
$$

and

$$
\begin{aligned}
& c+y \succ c+y^{\prime}, N\left(c^{\prime}, z\right)=N(c, y), \\
& \text { and } N\left(c^{\prime}, z^{\prime}\right)=N\left(c, y^{\prime}\right) \\
& \Rightarrow c^{\prime}+z \succ c^{\prime}+z^{\prime} .
\end{aligned}
$$

An agent exhibits a benchmark neural response if some null reaction occurs whenever $y=0$, i.e., there is an element $\mathbf{0}$ of Range $N$ such that, for all $c, N(c, 0)=\mathbf{0}$. We do not have to assume explicitly that a benchmark exists, but its presence would fit with our interpretation of $N$. The plausibility of (1) and (2) depends on how many increments $y$ may be added to $c$; if $y$ is a continuous variable, an agent may not display the required neurological discrimination.
The neural indicators can be ordered by the agent's ordinal ranking of consumption bundles: we define the relation $P_{0}$ on Range $N$ by $\eta P_{0} \eta^{\prime}$ if and only if there exist $c, y, y^{\prime}$ such that $\eta=N(c, y), \eta^{\prime}=N\left(c, y^{\prime}\right)$, and $c+y \succ c+$ $y^{\prime}$. Let $P$ be the transitive closure of $P_{0}$; we assume that $P$ is a weak order. Given $P$, equivalence classes of neural indicators in Range $N$ are defined by $\eta I \eta^{\prime} \Leftrightarrow$ not $\eta P \eta^{\prime}$ and not $\eta^{\prime}$ $P \eta .^{5}$ Notice that the ordering $P$ can be deduced solely from the observables $\succ$ and $N$.

If $P$ can be represented by a "utility" function $\Delta$, this function orders neural indicators according to whether the underlying consumption increments are more or less preferred: $\Delta(\eta)>$ $\Delta\left(\eta^{\prime}\right)$ if and only if $\eta P \eta^{\prime}$. Call any such $\Delta$ admissible.

Even if it turned out that $N$ maps into a single-dimensional variable that monotonically indicates which consumption increments are more preferred, it would not follow that the changes in satisfaction from increments to consumption are proportional to, or an affine transformation of $N(c, \cdot)$. (Indeed it would not matter if $N$ were a linear function of consumption, as Knutson and Peterson (2005) claim is the case.) Changes in satisfaction could well be formed by a differentand unobservable-psychological process and could as easily be the cube or exponential of $N(c$, - ) as an affine transformation of $N(c, \cdot)$. So, for the same reason that traditional ordinal utilities are arbitrary up to an increasing transformation, if $\Delta(N(c, \cdot))$ represents an agent's ordering of consumption increments given $c$, then any increasing transformation of $\Delta(N(c, \cdot))$ represents the same ordering equally well.

But to test for concavity, the arbitrariness of $\Delta$ is irrelevant; all that matters is the underlying $N$. Let us say that observational DMU obtains if, for any $c$ and $y$ and any admissible $\Delta$,

$$
\Delta(N(c+y, y)) \leq \Delta(N(c, y)) .
$$

If we assume that any equivalence class of neural indicators indicates a comparable subjective experience of well-being at any reference consumption bundle, then observational DMU

\footnotetext{
${ }^{5}$ Formally, $P$ is defined by $\eta P \eta^{\prime}$ if and only if $\eta P_{0} \eta^{\prime}$ or there is finite set $\left\{\eta_{1}, \ldots, \eta_{n}\right\}$ with $\eta P_{0} \eta_{1} P_{0} \cdots P_{0} \eta_{n} P_{0}$ $\eta^{\prime}$. To derive the conclusion that $P$ is a weak order, it is sufficient to assume that $P_{0}$ is acyclic and that if $\eta P \eta^{\prime} I \bar{\eta}$ or $\eta I \eta^{\prime} P \bar{\eta}$ then $\eta P \bar{\eta}$.
} 
means that a second increment of $y$ leads to a smaller psychic gain than the first increment of $y$. Since every admissible $\Delta$ represents the same ordering of neural indicators, if the inequality above holds for one admissible $\Delta$, then it holds for all admissible $\Delta$.

For an example of what observational DMU asserts, let there be just one consumption good, consumed at integer levels, and let the agent's preference relation $\succ$ always agree with $>$ (more is better). The neural responses are onedimensional. Specifically, we assume that for any $c$ the neural responses are linear in $y$ : for any $c$ there is a $k_{c}>0$ such that $N(c, y)=k_{c} y$ for all $y$. Evidently $P$ is the order $>$ (restricted to the integers). If in addition we assume that $c>c^{\prime}$ implies $k_{c}<k_{c^{\prime}}$, then observational DMU obtains. Notice that the linearity of each $N(c, \cdot)$ is consistent with observational DMU. Indeed observational DMU can be consistent with each $N(c, \cdot)$ having an increasing first derivative.

Given our interpretation that $N$ gives comparable information about changes in well-being or utility at all $c$ bundles, a psychology $\mathbf{U}$ for the agent should contain only those functions that represent those changes in utility. That is, if $\Delta(N(c, y)) \geq \Delta\left(N\left(c^{\prime}, y^{\prime}\right)\right)$ then a $u \in \mathbf{U}$ should satisfy $u(c+y)-u(c) \geq u\left(c^{\prime}+y^{\prime}\right)-u\left(c^{\prime}\right)$. So if observational DMU holds and $(c+y, y)$ and $(c, y)$ are in the domain of $N$, a $u \in \mathbf{U}$ should satisfy

$$
\frac{u(c+2 y)-u(c+y)}{u(c+y)-u(c)} \leq 1,
$$

or, in other words, $u$ is midpoint concave on its domain. (If $u$ satisfies midpoint concavity on a convex domain and is continuous, then $u$ is concave.)

Concavity of a psychology therefore leads to testable empirical claims. In some limiting cases, cardinality is testable too. By comparing $N(c, y)$ to $N\left(c^{\prime}, y^{\prime}\right)$, we may see if an agent regards a move from $c$ to $c+y$ as leading to a bigger or smaller change in satisfaction or utility than a move from $c^{\prime}$ to $c^{\prime}+y^{\prime}$. Hence, if every feasible increment $y$ is observable for all $c$, we can assemble from $N$ a complete ordering of how an agent compares changes in satisfaction. If in addition there is some utility $u$ that represents these changes whose range is an in- terval (e.g., when the consumption set is connected and $u$ is continuous) then the ordering of changes in satisfaction is sufficient to pin down a cardinal family of utilities (see Kaushik Basu, 1982). Determining whether a subject's neural responses satisfy the conditions we have placed on $N$ and whether some $u$ that represents the resulting ordering of changes in utility has an interval range would therefore constitute a test of cardinality. Not surprisingly, such a test is more demanding than a test for concavity. If only a discrete set of $(c, y)$ are in the domain of $N$ (as in our earlier example), then one cannot confirm or falsify the range condition. And if the variables $c$ and $y$ can be varied continuously it may be that the conditions (1) and (2) on $N$ no longer hold, again preventing a cardinality test.

Although we have so far used neural indicators to rank the satisfaction of consumption increments, the same theory applies to agents' verbal assessments of their satisfaction or happiness: simply interpret $N(c, y)$ as a onedimensional variable that reports an agent's assessment of the value of increment $y$ given the base $c$. When $N(c, y)>N\left(c^{\prime}, y^{\prime}\right)$, the agent says in effect that the utility gain of increment $y$ at base $c$ is greater than the utility gain of increment $y^{\prime}$ at base $c^{\prime}$. The neuroscience literature in fact finds that self-reported satisfaction and neural indicators correlate (see, e.g., Knutson et al., 2001). With regard to tests of cardinality, notice that the surveys of reported happiness levels allow agents to report only a few possible satisfaction responses; thus the interval range condition could not be even approximately verified.

When faced with neurological correlates of satisfaction or surveys of self-reported happiness, economists sometimes view these data as cardinal scales (e.g., Ng, 1997). I have deviated from this practice first by letting the set of neural indicators be arbitrary rather than a unidimensional variable. But even if $N$ is a single variable that correlates perfectly with happiness, our framework allows an agent's subjective sense of satisfaction to vary nonlinearly with $N$ (even when the interval range condition is satisfied and cardinality is testable). Regressions on survey data of self-reported happiness often import cardinality assumptions: if a regression treats equal differences between predicted and reported happiness as an equal loss of goodness of fit, the survey responses are 
being interpreted as cardinal scales. The use of compromise measurement scales would permit weaker and more plausible interpretations on survey data. When subjects compare increments of satisfaction, one could judge any deviation of a subject's ranking of satisfaction increments from a predicted ranking to be an equal goodness-of-fit failure. By making measurement assumptions explicit, it should be possible to free both physiological and self-reported satisfaction data from cardinality entanglements.

\section{Related Literature on Measurement}

Our ordering of properties of utility draws on two measurement literatures. The first is measurement theory proper (see, e.g., Krantz et al., 1971; Roberts, 1979; and, originally, Stanley S. Stevens, 1946), which identifies measurement classes with sets of transformations. Ratio scales are defined by the set of increasing linear transformations, interval or cardinal scales by the set of increasing affine transformations, and ordinal scales by the set of all increasing transformations. Measurement theory implicitly orders measurement classes by set inclusion; in the cases mentioned the sets of transformations are nested. A set inclusion ordering of transformations will sometimes track the ordering of properties we consider, but it covers only a few special cases and cannot define a sufficiently rich array of measurement classes. The second is the social choice literature that associates sets of transformations, now applied to multi-agent profiles of utility functions, with a category of interpersonal comparability (Amartya Sen, 1970; d'Aspremont and Gevers, 1977; Roberts, 1980b; Walter Bossert and John A. Weymark, 1996). Applying a smaller set of transformations imposes a tighter interpersonal comparability requirement.

The drawback of both literatures is that they either work directly with transformations instead of utility functions or, when sets of utilities are primitive, concentrate on those sets of utilities that are generated by applying a set (indeed a group) of transformations to an arbitrary utility function. At first glance, this practice may seem to be an advantage: any utility function can then be a member of any of the standard measurement classes. But taking arbitrary psychologies as primitive admits a greater variety of measurement standards and proves more flexible. For instance, the set of continuous utility functions defines a measurement standard that cannot be characterized by the set of continuous transformations (since an increasing continuous transformation applied to a noncontinuous utility function will not generate a continuous utility). Just as importantly, it is only by taking psychologies as primitive that we can identify the measurement requirements of assumptions on utility functions and hence to compare the measurement requirements of different assumptions.

Basu (1980) has also explored room for compromise between ordinal and cardinal utility theory. In a spirit similar to the present paper, Basu contends that DMU resides in this middle ground and remarks on the advantages of taking nonordinal assumptions as primitive. But Basu sticks to the method of characterizing measurement classes via utility transformations. Furthermore, as Basu (1982) shows, the middle ground that Basu (1980) linked to DMU ends up being equivalent to full-scale cardinality for continuous utilities defined on classical commodity spaces. Basu concludes that utility theory prior to the ordinal revolution used assumptions that were tantamount to cardinality (even when, as in Lange's case, they were attempting to rid themselves of cardinal assumptions). We have come to a different conclusion: by using sets of utility functions to compare measurement standards, compromises between cardinality and ordinality will persist even with standard economic commodities.

In contrast to the present view of measurement (and to the social choice view), the hardline ordinalist position has had trouble figuring out what to do with cardinal utility theories. A lot of work (e.g., Debreu, 1960; Krantz et al., 1971) has gone into finding axioms on preferences that ensure that preferences can be represented by functions that are unique up to increasing affine transformations. But from the ordinal vantage point, the significance of these representation results remains limited. Since preferences are the real primitive, the cardinal utility whose existence is established amounts only to a convenience. A von Neumann-Morgenstern utility function, for instance, simply allows the ease of a function that is linear in the probabilities. But other utility representations of the same preferences-e.g., the cube of a vNM utility - carry the same information about choice 
behavior. When psychologies are primitive, on the other hand, the cardinality of an assumption delivers the important message that the assumption entails a sizable departure from ordinality. Other nonordinal properties, such as concavity or continuity, imply a smaller departure.

\section{Conclusion}

The ordering of properties in this paper gauges the measurement requirements of assumptions on utility functions. To illustrate how this gauge can be applied, we argued in section III that the Arrow-Koopmans explanation of convexity, despite its billing as an ordinal theory, relies on a demanding cardinal standard of measurement rather than the weaker standard that concavity entails. Consider the following rebuttal, however. Although the Arrow-Koopmans theory begins with a class of integral utility functions, one could object that those integral utilities have no special significance; other utility functions that do not have the integral form represent the same preference relation as the integral utilities, and we could include them in the psychology that describes the agent. Furthermore, one can impose assumptions on preference relations that imply the existence of an integral utility representation (Grodal and Jean-François Mertens, 1968; Vind, 1969)just as there are axioms on preferences over finite numbers of goods that imply that additively separable utility representations exist (Debreu, 1960). So perhaps one could claim that the Arrow-Koopmans theory does give an ordinal explanation of the convexity of preferences. But this argument hinges on whether such an ordinal axiomatization stands as a convincing primitive. The key assumption needed for the existence of an integral utility representation is an independence postulate, just as a similar independence condition underlies the existence of additively separable utility representations. In the setting of time-dated goods, independence asserts that preferences over any subset of the time-dated goods are not affected by the consumption level of goods with different dates. Although independence is posed as an ordinal axiom, it rests on deeper psychological foundations: independence relies on a claim that consumption at one date does not affect the satisfaction of consumption at other dates. Indeed, this would appear to be the only rationale that could make independence plausible. But then only those functions where the utility experienced at one date is independent of the consumption at other dates will represent the full gamut of psychological claims asserted in an independence-based theory. As we know, the utility functions with this property form a cardinal psychology of additively separable functions (or in the infinite case, integral functions); the other ordinally equivalent functions do not express the psychological presuppositions that justify independence. This paper takes sets of utilities as primitive partly in order to give psychological rationales for ordinal axioms. Regarding convexity, it would beg the question to replace convexity with a different set of ordinal axioms that lead to the Arrow-Koopmans theory; one would then have to give rationales for those axioms. And the psychologies that accompany the rationales for some of those axions would be cardinal.

Orderings of properties of utility can shed light on the measurement requirements of rationales for other assumptions on preferences besides convexity. Consider, for example, continuity as an assumption on preferences (i.e., the assumption that strict upper and lower contour sets are open). The obvious justification for the continuity of preferences is to argue that satisfaction is a continuous psychological quantity. Although not quite ordinal, continuity of utility is weaker in the measurement sense than several other assumptions we have considered (e.g., additive separability, concavity on open sets). Thus, as intuition suggests, the continuity of preferences can be justified using a measurement standard only slightly stronger than ordinal measurement. Once again, an ordinalist might object that there is no need to assume that utility functions are continuous in order to give a rationale for preference continuity; the continuity of preferences already stands as an ordinal axiom. But the psychology that motivates an assumption that preferences are continuous is nonordinal; it turns on the claim that satisfaction is a continuous quantity.

If any doubt lingers that nonordinal content can motivate an axiom on preferences, consider the following trivial ordinalization of properties of utility. For any property $P$, define the property $P_{O}$ to consist of all functions that ordinally agree with some $u$ that satisfies $P$. Although $P_{O}$ evidently must be ordinal, the psychological 
theory that leads one to believe that $P$ obtains evidently need not be. Since the psychological theory behind independence or preference continuity also leads to a nonordinal psychology, the rebuttals we have been considering involve just this sort of trivial ordinalization.

\section{APPENDIX}

\section{PROOF OF THEOREM 1:}

If $\succeq$ is complete in addition to transitive and order-dense, the proof that there exists a $\mathbf{U}$ with $\succeq_{\mathbf{U}}=\succeq$ is the standard existence theorem for utility functions. So assume that $\succeq$ is not complete. Let $X / \sim$ denote the indifference classes of $\succeq: X / \sim$ is the partition of $X$ such that, for all $x \in$ $X$ and $y \in X,\{x, y\} \subset I \in X / \sim$ if and only if $x \succeq y$ and $y \succeq x$. Define $\succ$ on $X / \sim$ by $I \succ J$ if and only if $I \neq J$ and $x \succeq y$ for some $x \in I$ and $y \in$ $J$. For any $z \in X$, let $I(z)$ denote the indifference class that $z$ belongs to. For any $x, y \in X$ such that neither $x \succeq y$ nor $y \succeq x$ obtains, define two strict partial orders $\succ_{x}$ and $\succ_{y}$ on $X / \sim$ by $\succ U$ $\{(I(x), I(y))\}$ and $\succ \cup\{(I(y), I(x))\}$, respectively. Let $\succ_{x}^{t}$ and $\succ_{y}^{t}$ denote the transitive closures of $\succ_{x}$ and $\succ_{y}$, respectively.

Given a binary relation $P$ on domain $Z$, we say $T \subset Z$ is countably order-dense for $P$ if $T$ is countable, and for all $I, J \in Z \backslash T$ with $I P J$, there is a $K \in T$ such that $I P K P J$. Then, since $\succeq$ is countably order-dense, there is a $W \subset X / \sim$ that is countably order-dense for $\succ$ : one takes a countable $Y \subset X$ given by the countable orderdensity of $\succeq$ and sets $I \in W$ if and only if $I \cap$ $Y \neq \phi$. Let $W^{\prime}=W \cup\{I(x), I(y)\}$. To show that $W^{\prime}$ is countably order-dense for both $\succ_{x}^{t}$ and $\succ_{y}^{t}$, suppose, to take the case of $\succ_{x}^{t}$, that $I, J \in$ $X / \sim \backslash W^{\prime}$ satisfy $I \succ_{x}^{t} J$. If $I \succ_{x} J$ then $I \succ J$ and so, since $W$ is countably order-dense for $\succ$, there is a $K \in W^{\prime}$ such that $I \succ K \succ J$. If not $I$ $\succ_{x} J$, then by the definition of a transitive closure, there exists a finite set of indifference classes $\left\{I_{1}, \ldots, I_{n}\right\}$ such that $I \succ_{x} I_{1} \succ_{x} \cdots \succ_{x} I_{n}$ $\succ_{x} J$. If neither $I(x)$ nor $I(y)$ is in $\left\{I_{1}, \ldots, I_{n}\right\}$, then the transitivity of $\succ$ implies $I \succ J$, contradicting not $I \succ_{x} J$. If one of $I(x)$ or $I(y)$ appears in $\left\{I_{1}, \ldots, I_{n}\right\}$, then the transitivity of $\succ$ implies either $I \succ I(x) \succ J$ or $I \succ I(y) \succ J$ and both $I(x)$ and $I(y)$ are in $W^{\prime}$. If both $I(x)$ and $I(y)$ are in $\left\{I_{1}, \ldots, I_{n}\right\}$, then the transitivity of $\succ$ implies (1) $I \succ I(x) \succ J$, (2) $I \succ I(y) \succ J$, (3) $I \succ I(y) \succ_{x}$ $I(x) \succ J$, or (4) $I \succ I(x) \succ_{x} I(y) \succ J$. Cases (1) and (2) have already been covered, (3) cannot obtain since not $y \succeq x$, while in case (4) $I \succ I(x)$ $\succ_{x}^{t} J$. We may therefore apply Peter C. Fishburn (1979, Theorem 3.2) or Marcel K. Richter (1966) to conclude there is a utility function $u_{x, y}$ on $X / \sim$ such that $L \succ_{x}^{t} M$ implies $u(L)>u(M)$. Or, to argue directly, simply let $\varphi: W^{\prime} \rightarrow \mathbb{N}$ be one-to-one and define $u_{x, y}$ by

$$
u_{x, y}(I)=\sum_{J \in W^{\prime}: I \succ_{x}^{t} J} \frac{1}{2^{\varphi(J)}}
$$

for all $I \in X / \sim$ (letting a sum over the empty set equal 0$)$. Similarly, there exists a $u_{y, x}$ on $X / \sim$ such that $L \succ_{y}^{t} M$ implies $u(L)>u(M)$. Define the utility functions $v_{x, y}$ and $v_{y, x}$ on $X$ by setting $v_{x, y}(z)=u_{x, y}(I(z))$ and $v_{y, x}(z)=$ $u_{y, x}(I(z))$ for all $z \in X$.

Let $\mathbf{U}$ be defined by $v \in \mathbf{U}$ if and only if $v \in$ $\left\{v_{x, y}, v_{y, x}\right\}$ for some $x, y \in X$ such that not $x \succeq$ $y$ and not $y \succeq x$. Since $v_{x, y}(x)>v_{x, y}(y)$ and $v_{y, x}(y)>v_{y, x}(x)$, we have not $x \succeq_{\mathbf{U}} y$ and not $y$ $\succeq_{\mathbf{U}} x$. Since in addition it is easy to verify that $w \succeq z$ if and only if $u(w) \geq u(z)$ for all $u \in \mathbf{U}$, we have $\succeq_{\mathbf{U}}=\succeq$.

\section{PROOF OF THEOREM 3:}

To show that concavity is no stronger than any cardinal property, let $\mathbf{U}_{C}$ be cardinal, let $\mathbf{U}_{C V}$ maximally satisfy concavity, and suppose there exists $u \in \mathbf{U}_{C} \cap \mathbf{U}_{C V}$. Since $u \in \mathbf{U}_{C}$, for any $u^{\prime} \in \mathbf{U}_{C}$ there exists an increasing affine transformation $g$ such that $g \circ u=u^{\prime}$. Since an increasing affine transformation of a concave function is concave, $u^{\prime} \in \mathbf{U}_{C V}$, and so $\mathbf{U}_{C V} \supset$ $\mathbf{U}_{C}$. We omit the equally elementary proof that any ordinal property is no stronger than concavity (or indeed no stronger than any property). For result (ii), let the cardinal property now have range $>2$, and suppose $v \in \mathbf{U}_{C} \cap \mathbf{U}_{C V}$. If $f$ : $\mathbb{R} \rightarrow \mathbb{R}$ is increasing and strictly concave, we have, since $v \in \mathbf{U}_{C V}, f \circ v \in \mathbf{U}_{C V}$. Since $\mid$ Range $v \mid$ $>2$, there exist $v_{1}, v_{2}, v_{3} \in$ Range $v$ and $\lambda \in(0$, 1) with $v_{1}<v_{2}<v_{3}$ and $v_{2}=\lambda v_{1}+(1-\lambda) v_{3}$. Since $f$ is strictly concave, $f\left(v_{2}\right)>\lambda f\left(v_{1}\right)+(1-$ ג) $f\left(v_{3}\right)$. So $f \mid$ Range $v$ is not affine and therefore $f$ $\circ v \notin \mathbf{U}_{C}$. Hence concavity is strictly weaker than any cardinal property with range $>2$ that intersects concavity.

To establish (iii), let $\mathbf{U}_{O}$ maximally satisfy an ordinal property with range $>1$, let $\mathbf{U}_{C V C}$ maximally satisfy concavity and continuity, and suppose that $\mathbf{U}_{O} \cap \mathbf{U}_{C V C} \neq \varnothing$. For $v \in \mathbf{U}_{O} \cap$ 
$\mathbf{U}_{C V C}$, Range $v$ is a nontrivial interval. So let $x_{1}$, $x_{2} \in X$ satisfy $v\left(x_{1}\right)<v\left(x_{2}\right)$ and define $D=$ $\left\{w \in X: w=\lambda x_{1}+(1-\lambda) x_{2}\right.$ for some $\lambda \in$ $[0,1]\}$. Since $v$ is concave there is a $C \subset D$ that is connected and such that $v$ is monotone on $C$. So there are $z_{1}, z_{2}, z_{3} \in C$ that satisfy $z_{3}=(1 / 2) z_{1}+(1 / 2) z_{3}$ and $v\left(z_{1}\right)<v\left(z_{2}\right)<$ $v\left(z_{3}\right)$. Let $g: \mathbb{R} \rightarrow \mathbb{R}$ be an increasing transformation such that $g\left(v\left(z_{2}\right)\right)<(1 / 2) g\left(v\left(z_{1}\right)\right)+$ $(1 / 2) g\left(v\left(z_{3}\right)\right)$. We then have $g \circ v \notin \mathbf{U}_{C V C}$, but since $g$ is increasing, $g \circ v \in \mathbf{U}_{O}$.

\section{PROOF OF THEOREM 4:}

See Grodal (1974).

\section{PROOF OF THEOREM 5:}

Let $\mathbf{U}$ with domain $\mathcal{L}$ maximally satisfy utility integrability and let $U$ be an arbitrary element of $\mathbf{U}$. Since it is plain that if $W: \mathcal{L} \rightarrow \mathbb{R}$ is such that there exists an increasing affine transformation $g$ that satisfies $g \circ U=W$, then $W$ satisfies utility integrability, we need only show that for any $W \in \mathbf{U}$ there exists an increasing affine transformation $g$ such that $W=g \circ U$. It is sufficient to show that if $g$ is an increasing transformation and $W=g \circ U$ satisfies utility integrability (i.e., $W \in \mathbf{U}$ ), then $g$ is affine.

We establish this via Theorem 2. First, observe that since $\mid$ Range $U \mid>1$, there exist $\alpha, \alpha^{\prime}$ $\in \mathcal{L}$ such that $U(\alpha)>U\left(\alpha^{\prime}\right)$, and hence there also exists, for any $\varepsilon>0$, a measurable $C_{1} \subset$ $[0, T]$ such that

$$
0<\int_{C_{1}}\left(u(\alpha(t), t)-u\left(\alpha^{\prime}(t), t\right)\right) d \mu(t)<\varepsilon
$$

where, for $U \in \mathbf{U}, u: \mathbb{R}_{+}^{n} \times[0, \mathrm{~T}] \rightarrow \mathbb{R}$ is one of the functions satisfying Definition 10 (i). (This $u$ is fixed throughout.) By setting $\varepsilon$ sufficiently small, we can partition $[0, T]$ into sets $C_{1}$ and $C_{2}$ such that in addition

$$
\int_{C_{2}}\left(u(\alpha(t), t)-u\left(\alpha^{\prime}(t), t\right)\right) d \mu(t)>0 .
$$

For $i=1,2$, let $\mathcal{L}_{i}$ be the restriction of $\mathcal{L}$ to $C_{i}$ - that is, the set of functions from $C_{i}$ to $\mathbb{R}^{n}$ defined by $\alpha_{i} \in \mathcal{L}_{i}$ if and only if there exists $\alpha \in \mathcal{L}$ such that $\alpha_{i}(t)=\alpha(t)$ for all $t \in C_{i}$.
Let $U_{i}: \mathcal{L}_{i} \rightarrow \mathbb{R}$ be defined by $U_{i}\left(\alpha_{i}\right)=$ $\int_{C_{i}} u\left(\alpha_{i}(t), t\right) d \mu(t)$. We have $\mathcal{L}=\mathcal{L}_{1} \times \mathcal{L}_{2}$. Using $\left(\alpha_{1}, \alpha_{2}\right)$ to denote the $\alpha \in \mathcal{L}$ such that $\alpha(t)=\alpha_{i}(t)$ for $t \in C_{i}$ and $i \in\{1,2\}$, we have $U\left(\alpha_{1}, \alpha_{2}\right)=U_{1}\left(\alpha_{1}\right)+U_{2}\left(\alpha_{2}\right)$ for all $\alpha \in \mathcal{L}$. Since, for $i \in\{1,2\}, U_{i}\left(\alpha_{i}\right)>$ $U_{i}\left(\alpha_{i}^{\prime}\right)$, $\mid$ Range $U_{i} \mid>1$.

To show that the $U_{i}$ have ranges equal to nontrivial intervals, allowing us to apply Theorem 2, we use the following result from the theory of integration of correspondences, sometimes called Lyapunov's theorem.

LYAPUNOV'S THEOREM: Given an atomless measure space $(\Omega, \mathcal{F}, \lambda)$ and correspondence $P: \Omega \rightrightarrows \mathbb{R}$, the set $\int_{\Omega} P d \lambda \equiv\left\{\int_{\Omega} p d \lambda\right.$ : $p$ is integrable and $p(\omega) \in P(\omega)$ for a.e. $\omega \in \Omega\}$ is convex.

By Lyapunov's theorem, Range $U$, Range $U_{1}$, and Range $U_{2}$ are convex sets and therefore intervals. For example, for the case of Range $U_{1}$, the measure space is Lebesgue measure on $C_{1}$ and the correspondence $P$ would be defined by $P(t)=\left\{u\left(\alpha_{1}(t), t\right) \in \mathbb{R}: \alpha_{1} \in \mathcal{L}_{1}\right\}$ for each $t \in C_{1}$. Since Range $U_{1}=\int_{C_{1}} P d \mu$, we conclude that Range $U_{1}$ is convex and hence an interval. Note that since $\mid$ Range $U_{1} \mid>1$ and $\mid$ Range $U_{2} \mid>1$, Range $U_{1}$ and Range $U_{2}$ have nonempty interiors.

Since $W \in \mathbf{U}$, there exists a $w: \mathbb{R}_{+}^{n} \times[0, T] \rightarrow$ $\mathbb{R}$, where $t \mapsto w(\alpha(t), t)$ is integrable and $W(\alpha)=$ $g(U(\alpha))=\int_{0}^{T} v(\alpha(t), t) d \mu(t)$ for all $\alpha \in \mathcal{L}$. Just as we argued for the case of $U, U_{1}$, and $U_{2}$, we have $W\left(\alpha_{1}, \alpha_{2}\right)=W_{1}\left(\alpha_{1}\right)+W_{2}\left(\alpha_{2}\right)$ for all $\left(\alpha_{1}\right.$, $\left.\alpha_{2}\right) \in \mathcal{L}$, where $W_{i}\left(\alpha_{i}\right)=\int_{C_{i}} w\left(\alpha_{i}(t), t\right) d \mu(t)$, $i \in\{1,2\}$. Hence $g\left(U_{1}\left(\alpha_{1}\right)+U_{2}\left(\alpha_{2}\right)\right)=$ $W_{1}\left(\alpha_{1}\right)+W_{2}\left(\alpha_{2}\right)$ for all $\left(\alpha_{1}, \alpha_{2}\right) \in \mathcal{L}$. So apply Theorem 2 to conclude that $g$ is affine.

\section{PROOF OF THEOREM 6:}

In text. See also the proof of Theorem 3.

\section{PROOF OF THEOREM 7:}

We omit the details: $7(i)$ is obvious and 7 (ii) is a slight variant on common arguments in the social choice literature (see, e.g., Moulin, 1988).

\section{PROOF OF THEOREM 8:}

If $\mathbf{v} \in \operatorname{con}\left\{\mathbf{u} \in F(\bar{u}): \mathbf{u}^{*} \geq \mathbf{w}^{*}\right\}$ then there exist $\lambda^{1}, \ldots, \lambda^{m} \in[0,1]$ and $\mathbf{z}^{1}, \ldots, \mathbf{z}^{m} \in F(\bar{u})$ such that $\sum_{k=1}^{m} \lambda^{k}=1, \mathbf{v}=\sum_{k=1}^{m} \lambda^{k} \mathbf{z}^{k}$, and 
$\mathbf{z}^{k *} \geq \mathbf{w}^{*}$ for $k=1, \ldots, m$. For any increasing $g$ : Range $\bar{u} \rightarrow \mathbb{R}$ such that $g \mid \bar{u}(i, X)$ is concave for each $i \in \mathbb{\square}$,

$$
\begin{aligned}
& \sum_{i \in \mathbb{\Xi}} g\left(\mathbf{v}_{i}\right)=\sum_{i \in \mathbb{\Xi}} g\left(\sum_{k=1}^{m} \lambda^{k} \mathbf{z}_{i}^{k}\right) \geq \sum_{i \in \mathbb{I}}\left[\sum_{k=1}^{m} \lambda^{k} g\left(\mathbf{z}_{i}^{k}\right)\right] \\
& =\sum_{k=1}^{m} \lambda^{k}\left[\sum_{i \in \rrbracket} g\left(\mathbf{z}_{i}^{k}\right)\right]=\sum_{k=1}^{m} \lambda^{k}\left[\sum_{i \in \rrbracket} g\left(\mathbf{z}_{i}^{k *}\right)\right] \\
& \geq \sum_{k=1}^{m} \lambda^{k}\left[\sum_{i \in \mathbb{l}} g\left(\mathbf{w}_{i}^{*}\right)\right] \\
& =\sum_{k=1}^{m} \lambda^{k}\left[\sum_{i \in \mathbb{I}} g\left(\mathbf{w}_{i}\right)\right] \\
& =\sum_{i \in \mathbb{I}}\left[\sum_{k=1}^{m} \lambda^{k} g\left(\mathbf{w}_{i}\right)\right]=\sum_{i \in \mathbb{I}} g\left(\mathbf{w}_{i}\right),
\end{aligned}
$$

where the two inequalities follow respectively from the concavity and increasingness of each $g \mid \bar{u}(i, X)$. Since $\mathbf{v}, \mathbf{w} \in F(\bar{u})$, there exist $x, y \in$ $X$ such that $\mathbf{v}=(\bar{u}(1, x), \ldots, \bar{u}(I, x))$ and $\mathbf{w}=$ $(\bar{u}(1, y), \ldots, \bar{u}(I, y))$. Since $\bar{u}$ is lcc, for each $u \in$ $\mathbf{U}_{C C}$ there is a $g$ meeting the above assumptions such that $u=g \circ \bar{u}$. Hence $\sum_{i \in \mathbb{}} u(i, x) \geq \sum_{i \in \mathbb{D}}$ $u(i, y)$ for all $u \in \mathbf{U}_{C C}$ and therefore $x \succeq_{\mathbf{U}_{C C}}^{s} y$ and $\mathbf{v} R_{\bar{u} \in \mathbf{U}_{C C}} \mathbf{w}$.

\section{PROOF OF THEOREM 9:}

For $\mathbf{y} \in \mathbb{R}^{I}$, let $\mathbf{y}_{\max }$ denote $\max \left\{\mathbf{y}_{j}, \mathbf{y}_{k}\right\}$ and $\mathbf{y}_{\text {min }}$ denote $\min \left\{\mathbf{y}_{j}, \mathbf{y}_{k}\right\}$.

Assume $\mathbf{v}_{j}+\mathbf{v}_{k} \geq \mathbf{w}_{j}+\mathbf{w}_{k}$ and $\mathbf{v}_{\text {min }} \geq \mathbf{w}_{\text {min }}$. If both $\mathbf{v}_{j}+\mathbf{v}_{k}=\mathbf{w}_{j}+\mathbf{w}_{k}$ and $\mathbf{v}_{\text {min }}=\mathbf{w}_{\text {min }}$ then $\mathbf{v}^{*}=\mathbf{w}^{*}$ and the conclusion $\mathbf{v} R_{\bar{u} \in \mathbf{U}_{C C}} \mathbf{w}$ is immediate. So assume at least one inequality is strict. Define $\mathbf{z}^{1} \in \mathbb{R}^{I}$ by $\mathbf{z}_{j}^{1}=\mathbf{w}_{\min }, \mathbf{z}_{k}^{1}=\mathbf{v}_{j}+$ $\mathbf{v}_{k}-\mathbf{w}_{\min }$, and $\mathbf{z}_{i}^{1}=\mathbf{v}_{i}$ for $i \notin\{j, k\}$ and let $\mathbf{z}^{2}$ equal $\mathbf{z}^{1}$ with the $j$ and $k$ coordinates interchanged. Since $\mathbf{v}_{j}+\mathbf{v}_{k} \geq \mathbf{w}_{j}+\mathbf{w}_{k}$, we conclude that $\mathbf{z}_{k}^{1} \geq \mathbf{w}_{\max }$ and therefore $\mathbf{z}^{*} \geq \mathbf{w}^{*}$ and $\mathbf{z}^{2 *} \geq \mathbf{w}^{*}$. Since either $\mathbf{v}_{j}+\mathbf{v}_{k}>\mathbf{w}_{j}+\mathbf{w}_{k}$ or $\mathbf{v}_{\text {min }}>\mathbf{w}_{\text {min }}$, the equality $\mathbf{v}_{j}=\lambda \mathbf{w}_{\text {min }}+(1-\lambda)$ $\left(\mathbf{v}_{j}+\mathbf{v}_{k}-\mathbf{w}_{\text {min }}\right)$ defines a $\lambda$ in $[0,1]$ and this $\lambda$ also solves $\mathbf{v}_{k}=\lambda\left(\mathbf{v}_{j}+\mathbf{v}_{k}-\mathbf{w}_{\text {min }}\right)+(1-\lambda) \mathbf{w}_{\text {min }}$. Hence $\mathbf{v}=\lambda \mathbf{z}^{1}+(1-\lambda) \mathbf{z}^{2}$.
Let $g$ be increasing and concave on Range $\bar{u}$ and extend $g$ to any $r$ such that $r>s$ for all $s \in$ Range $\bar{u}$ by setting $g(r)=\sup \{g(s): s \in$ Range $\bar{u}$ \}. Since $g$ remains concave and weakly increasing, the indented equalities and inequalities in the proof of Theorem 8 apply to such a $g$. To conclude that $\mathbf{v} R_{\bar{u} \in \mathbf{U}_{C C}} \mathbf{w}$, therefore, it is sufficient to show that for any $u \in \mathbf{U}_{C C}$ there is a $g$ : Range $\bar{u} \rightarrow \mathbb{R}$ that is increasing and concave on Range $\bar{u}$ such that $u=g \circ \bar{u}$. Since $\bar{u}$ is lcc there is an increasing $h$ : Range $\bar{u} \rightarrow \mathbb{R}$ with $u=$ $h \circ \bar{u}$ such that, for all $i \in \mathbb{}, h \mid \bar{u}(i, X)$ is concave. Since $u(i, X)$ and $\bar{u}(i, X)$ are both intervals and $h$ is increasing, $h \mid \bar{u}(i, X)$ is continuous. Also observe that since $\cup_{i \in \mathbb{n}}$ Int $\bar{u}(i, X)$ is an interval, Range $\bar{u}$ is an interval. Now for any $y \in$ Range $\bar{u}$, there is an $i$ and nontrivial interval $E$ with $y \in$ $E$ and $E \subset$ Int $\bar{u}(i, X)$. Since $h$ is concave and continuous on $E$, the left and right derivatives of $h$ are both nonincreasing on $E$ and the right derivative at any point in $E$ is less than or equal to the left derivative. These properties of the left and right derivatives therefore hold at every point in Range $\bar{u}$ which, together with the fact that Range $\bar{u}$ is an interval, implies that $h$ is concave.

Now assume $\mathbf{v} R_{\bar{u} \in \mathbf{U}_{C C}} \mathbf{w}$. The argument in this direction does not require that $\cup_{i \in \rrbracket}$ Int $\bar{u}(i, X)$ be an interval. If $\mathbf{v}_{j}+\mathbf{v}_{k}<\mathbf{w}_{j}+\mathbf{w}_{k}$, then $\sum_{i \in \mathbb{\square}}$ $\mathbf{v}_{i}<\sum_{i \in \mathbb{}} \mathbf{w}_{i}$, contradicting $\mathbf{v} R_{\bar{u} \in \mathbf{U}_{C C}} \mathbf{w}$. Hence $\mathbf{v}_{j}+\mathbf{v}_{k} \geq \mathbf{w}_{j}+\mathbf{w}_{k}$. Suppose, contrary to the theorem, that $\mathbf{v}_{\text {min }}<\mathbf{w}_{\text {min }}$. Consider transformations $g$ : con(Range $\bar{u}) \rightarrow \mathbb{R}$ that are piecewise-linear, increasing, concave, and whose only nondifferentiability occurs at $\mathbf{w}_{\text {min }}$. As long as $D g\left(u^{\prime}\right)>D g\left(u^{\prime \prime}\right)>0$ for $u^{\prime}<\mathbf{w}_{\min }$ and $u^{\prime \prime}>\mathbf{w}_{\min }, g$ will be concave and increasing, but otherwise these two derivatives are arbitrary. So fix some $D g\left(u^{\prime}\right)>0$ and choose $D g\left(u^{\prime \prime}\right)>0$ small enough that $g\left(\mathbf{v}_{\max }\right) \approx g\left(\mathbf{w}_{\max }\right)$. (Note that $\mathbf{v}_{\text {max }}>\mathbf{w}_{\text {min }}$ since $\mathbf{v}_{\text {min }}<\mathbf{w}_{\text {min }}$ and $\mathbf{v}_{j}+\mathbf{v}_{k} \geq$ $\mathbf{w}_{j}+\mathbf{w}_{k}$.) Hence $g\left(\mathbf{v}_{j}\right)+g\left(\mathbf{v}_{k}\right)<g\left(\mathbf{w}_{j}\right)+g\left(\mathbf{w}_{k}\right)$ and so $\sum_{i \in \llbracket} g\left(\mathbf{v}_{i}\right)<\sum_{i \in \mathbb{}} g\left(\mathbf{w}_{i}\right)$, again contradicting $\mathbf{v} R_{\bar{u} \in \mathbf{U}_{C C}} \mathbf{w}$.

\section{REFERENCES}

Arrow, Kenneth J. "An Extension of the Basic Theorems of Classical Welfare Economics," in Jerzy Neyman, ed., Proceedings of the second Berkeley Symposium on Mathematical Statistics and Probability. Berkeley: University of California Press, 1951, pp. 507-32. 
d'Aspremont, Claude and Gevers, Louis. "Equity and the Informational Basis of Collective Choice." Review of Economic Studies, 1977, 44(2), pp. 199-209.

Basu, Kaushik. Revealed preference of government. Cambridge, MA: Cambridge University Press, 1980.

Basu, Kaushik. "Determinateness of the Utility Function: Revisiting a Controversy of the Thirties." Review of Economic Studies, 1982, 49(2), pp. 307-11.

Bawa, Vijay S. "Optimal Rules for Ordering Uncertain Prospects." Journal of Financial Economics, 1975, 2(1), pp. 95-121.

Blackorby, Charles and Donaldson, David. "Utility vs. Equity: Some Plausible Quasi-Orderings." Journal of Public Economics, 1977, 7(3), pp. 365-81.

Blanchflower, David G. and Oswald, Andrew J. "Well-Being over Time in Britain and the USA." Journal of Public Economics, 2004, 88(7-8), pp. 1359-86.

Bossert, Walter and Weymark, John A. "Utility in Social Choice," in Salvador Barberá, Peter J. Hammond, and Christian Seidl, eds., Handbook of utility theory. Vol. 2. Dordrecht: Kluwer Academic Publisher, 2004, pp. 1099-1177.

Breiter, Hans C.; Aharon, Itzhak; Kahneman, Daniel; Dale, Anders and Shizgal, Peter. "Functional Imaging of Neural Responses to Expectancy and Experience of Monetary Gains and Losses." Neuron, 2001, 30(2), pp. 619-39.

Debreu, Gerard. "Topological Methods in Cardinal Utility Theory," in Kenneth J. Arrow, Samuel Karlin and Patrick Suppes, eds., Mathematical methods in the social sciences. Stanford: Stanford University Press, 1960, pp. 16-26.

Debreu, Gerard. "Least Concave Utility Functions." Journal of Mathematical Economics, 1976, 3(2), pp. 121-29.

Dixit, Avinash K. and Seade, Jesus. "Utilitarian versus Egalitarian Redistributions." Economics Letters, 1979, 4(2), pp. 121-24.

Dubra, Juan; Maccheroni, Fabio and Ok, Efe A. "Expected Utility Theory without the Completeness Axiom." Journal of Economic Theory, 2004, 115(1), pp. 118-33.

Easterlin, Richard A. "Does Economic Growth Improve the Human Lot? Some Empirical Evidence," in Paul A. David and Melvin W. Reder, eds., Nations and households in eco- nomic growth: Essays in honor of Moses Abramowitz. New York: Academic Press, 1974, pp. 89-125.

Edgeworth, Francis Y. "The Pure Theory of Taxation, III." Economic Journal, 1897, 7(28), pp. $550-71$.

Fishburn, Peter C. Utility theory for decision making. New York: R. E. Krieger Publishing Company, 1979.

Foster, James E. and Shorrocks, Anthony F. "Poverty Orderings." Econometrica, 1988a, 56(1), pp. 173-77.

Foster, James E. and Shorrocks, Anthony F. "Poverty Orderings and Welfare Dominance." Social Choice and Welfare, 1988b, 5(2/3), pp. 179-98.

Glimcher, Paul W. and Rustichini, Aldo. "Neuroeconomics: The Consilience of Brain and Decision." Science, 2004, 306(5695), pp. 44752.

Gorman, W. M. "Convex Indifference Curves and Diminishing Marginal Utility." Journal of Political Economy, 1957, 65(1), pp. 4050.

Grodal, Birgit. "Convexity of Preferences," in Jerzy Los and Maria W. Los, eds., Mathematical models in economics: Proceedings of the Symposium on Mathematical Methods of Economics, February-July 1972, and of the Conference on von Neumann Models, 10-15 July 1972. Amsterdam: North-Holland, 1974, pp. 441-48.

Grodal, Birgit and Mertens, Jean-François. "Integral Representation of Utility Functions." Center for Operations Research and Econometrics, CORE Discussions Papers: No. 6823, 1968.

Hammond, Peter J. "Equity, Arrow's Conditions, and Rawls' Difference Principle." Econometrica, 1976, 44(4). pp. 793-804.

Hanoch, Giora and Levy, Haim. "The Efficiency Analysis of Choices Involving Risk." Review of Economic Studies, 1969, 36(107), pp. 33546.

Knutson, Brian; Adams, Charles M.; Fong, Grace W. and Hommer, Daniel. "Anticipation of Increasing Monetary Reward Selectively Recruits Nucleus Accumbens." Journal of Neuroscience, 2001, 21(RC159), pp. 1-5.

Kolm, Serge-Christophe. "The Optimal Production of Social Justice," in Julius Margolis and Henri Guitton, eds., Public economics. London: Macmillan, 1969, pp. 145-200. 
Krantz, David H.; Luce, R. Duncan; Suppes, Patrick and Tversky, Amos. Foundations of measurement. Vol. I. New York: Academic Press, 1971.

Mandler, Michael. Dilemmas in economic theory: Persisting foundational problems of microeconomics. New York: Oxford University Press, 1999.

Mandler, Michael. "Compromises between Cardinality and Ordinality in Preference Theory and Social Choice." Cowles Foundation, Yale University, Cowles Foundation Discussion Papers: No. 1322, 2001.

Mandler, Michael. "Orderings of Properties of Utility Functions.” Unpublished Paper, 2003.

Mas-Colell, Andreu; Whinston, Michael D. and Green, Jerry R. Microeconomic theory. New York: Oxford University Press, 1995.

Moulin, Hervé. Axioms of cooperative decision making. Cambridge: Cambridge University Press, 1988.

Ng, Yew-Kwang. "A Case for Happiness, Cardinalism, and Interpersonal Comparability." Economic Journal, 1997, 107(445), pp. 1848-58.

Ok, Efe A. "Utility Representation of an Incomplete Preference Relation." Journal of Economic Theory, 2002, 104(2), pp. 42949.

Paulus, Martin P.; Hozack, Nikki; Zauscher, Blanca; McDowell, Jennifer E.; Frank, Lawrence; Brown, Gregory G. and Braff, David L.
"Prefrontal, Parietal, and Temporal Cortex Networks Underlie Decision-Making in the Presence of Uncertainty." Neuroimage, 2001, 13(1), pp. 91-100.

Pigou, Arthur C. Economics of welfare. 4th ed. London: Macmillan, 1932.

Richter, Marcel K. "Revealed Preference Theory." Econometrica, 1966, 34(3), pp. 635-45.

Roberts, Fred. Measurement theory with applications to decision making, utility, and the social sciences. Reading, MA: Addison-Wesley Publishing Company, 1979.

Roberts, Kevin W. S. "Interpersonal Comparability and Social Choice Theory." Review of Economic Studies, 1980a, 47(2), pp. 421-39.

Roberts, Kevin W. S. "Possibility Theorems with Interpersonally Comparable Welfare Levels." Review of Economic Studies, 1980b, 47(2), pp. 409-20.

Rustichini, Aldo, ed. "Special Issue on Neuroeconomics." Games and Economic Behavior, 2005, 52(2).

Sen, Amartya. "Interpersonal Aggregation and Partial Comparability." Econometrica, 1970, 38(3), pp. 393-409.

Shorrocks, Anthony F. "Ranking Income Distributions." Economica, 1983, 50(197), pp. 3-17.

Stevens, Stanley S. "On the Theory of Scales of Measurement." Science, 1946, 103(2684), pp. 677-80.

Vind, Karl. Mean groupoids. Copenhagen: University of Copenhagen, 1969. 